\title{
Modeling Discrete and Rhythmic Movements through Motor Primitives: A Review*
}

\author{
Sarah Degallier \\ Auke Ijspeert \\ Ecole Polytechnique Fédérale de Lausanne (EPFL), Suisse \\ sarah.degallier@epfl.ch
}

\begin{abstract}
Rhythmic and discrete movements are frequently considered separately in motor control, probably because different techniques are commonly used to study and model them. Yet, an increasing interest for a comprehensive model for movement generation requires to bridge the different perspectives arising from the study of those two types of movements. In this article, we consider discrete and rhythmic movement within the framework of motor primitives, i.e. of modular generation of movements. Thereby we hope to get an insight into the functional relationships between discrete and rhythmic movements and thus into a suitable representation for both of them. Within this framework we can define four possible categories of modeling for discrete and rhythmic movements depending on the required command signals and on the spinal processes involved in the generation of the movements. These categories are first discussed relatively to biological concepts such as force fields and central pattern generators and are then illustrated by several mathematical models based on dynamical system theory. A discussion on the plausibility of theses models concludes this work.
\end{abstract}

This article was originally published in the journal Biological Cybernetics. The final publication is available at www.springerlink.com: http: //www.springerlink. com/content/x7n0355642074wx0

* This work was supported by the European Commission's Cognition Unit, project no. IST-2004-004370: RobotCub and by the Swiss National Science Foundation.

\section{Introduction}

Humans are able to adapt their movements to almost any new situation in a very robust, seemingly effortless way. To explain both adaptivity and robustness, a very promising perspective is the modular approach to movement generation: Movements result from combinations of a finite set of stable motor primitives organized at the spinal level (see Bizzi et al (2008) for a review). In this article, a motor primitive is defined as a network of spinal neurons that activates a set of muscles (that we call a synergy) in a coordinated way in order to execute a specific movement. Motor primitives are thus defined relative to the movement that they produce.

In terms of control, the modularity assumption is attractive because it drastically reduces the dimensionality of the problem: instead of a complex stimulation of a vast number of muscles across the body, high-level commands can be summed up as activation signals for a finite, discrete set of motor primitives. Strong evidence, notably through the concepts of central pattern generators and force fields (see resp. reviews by Grillner (2006) and Bizzi et al (2008)), supports the existence of such functional modules at the spinal level in vertebrate animals. For instance, Kargo and Giszter (2000) have demonstrated how a finite set of spinal motor primitives could account for the natural wiping reflex in the frog, showing that the central nervous system (CNS) could use such primitives to produce natural behaviors.

Assuming the existence of such motor primitives provides an interesting framework for reflecting upon the potential differences between discrete and rhythmic movements. It allows us to reflect on these movements relatively to a simplified view of movement generation: a high level command activates a (set of) motor primitive(s) at the spinal level that generates a given kinematic outcome. Given this scheme, we can consider 
the potential differences between discrete and rhythmic movements that are not related to sensory feedback nor muscles interaction but to the spinal processes underlying them and to the high-level commands needed to activate these spinal processes. We call this approach a functional approach to distinguish it from the many studies focusing on the kinematics of these types of movements, as for instance the thorough analysis by Hogan and Sternad (2007).

Most of the studies on discrete and rhythmic movements are either based on electromyographic (EMG) analyses of the generated movements (Hogan and Sternad (2007), van Mourik and Beek (2004)) or on functional magnetic resonance imaging (fMRI) analysis (Schaal et al (2004)) as will be reviewed in Section 3. While those studies have provided insightful results on the nature of discrete and rhythmic movements, we think that adopting a functional perspective is a useful, complementary step to understand the differences between the movements regarding the way that they are generated, and also to gain more understanding on how brain and EMG studies can be bridged. Moreover, the generation of discrete and rhythmic movements at the spinal level has been extensively studied in vertebrates through the concepts of force fields and central pattern generators respectively, providing an interesting basis for reflection.

We start by presenting a simplified model of the motor system on which we will base our reflection (Section 2 ). We then present several studies on the differences between discrete and rhythmic movements (Section 3) and some of the literature on the combination of these movements (Section 4). Altough we are well aware that movement generation is a dynamic process involving the whole motor system, we discuss movement execution and movement planning separately since we think that in this way distinct properties pertaining to those two phases of movement can be emphasized, as will be discussed in Sections 5 and 6 respectively. Furthermore we present in Section 7 some existing mathematical models for the generation of discrete and rhythmic movement, since such models provide discerning information on the generation of these movements.

\section{A simplified view on motor sys- tems}

In this section, we briefly present a simple model for movement generation based on the concept of motor primitives. We consider the processes underlying the generation of both movements with an emphasis on the contribution of the spinal component of the CNS. Such a simplified structure will provide us with a framework of discussion throughout this article.

According to textbooks (see for instance Kandel et al (2000)), movement generation is achieved through three motor structures organized hierarchically and corresponding to different levels of abstraction. These structures are (a) the cerebral cortex, which is responsible for defining the motor task; (b) the brain stem, which elaborates the motor plan to execute the motor task; and (c) the spinal cord, which generates the spatiotemporal sequence of muscles activation to execute the task. In addition, the cerebral cortex and the brain stem are influenced by the cerebellum and the basal ganglia, which can be considered as feedback circuits, the cerebellum being also connected to the spinal cord.

In order to consider the relationships between discrete and rhythmic movements, we will mainly distinguish between the planning (a) and the execution phase (b-c) of movements. By planning, we mean all the processes required to choose the features of the movement (i.e. to represent the task) and by execution, the processes responsible for the spatio-temporal activation of the muscles generating the corresponding trajectories by the limbs. Within this framework, four different possible structures for the generation of discrete and rhythmic movements need to be considered (see Fig.1):

\section{Two/Two}

Discrete and rhythmic movements are generated through two totally different processes, both at the planning and the execution phase;

\section{One/Two}

The planning processes involved in the generation of both movements are the same, while their generation depends on different structures;

\section{One/One}

Discrete and rhythmic movements are two outcomes of the same process, both at the planning 
and the execution level;

\section{Two/One}

The two movements involve different types of representations, while the generator is common.

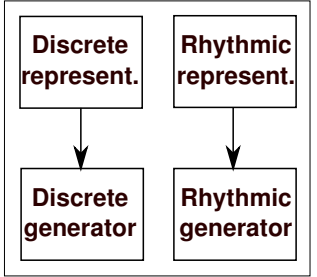

(a) Two/Two

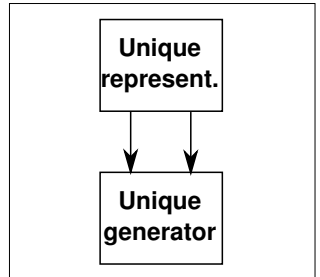

(c) One/One

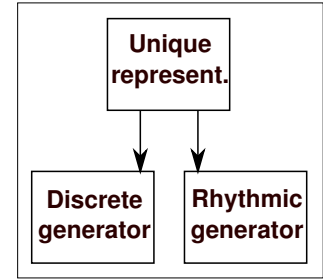

(b) One/Two

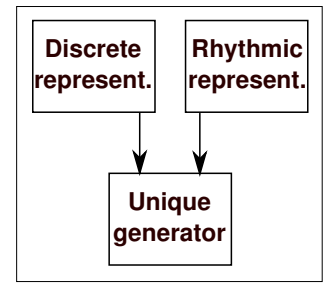

(d) Two/One
Figure 1: Schematic of the four different categories of models.

These four simple categories provide us with basic grounds for reflection on the possible differences between discrete and rhythmic movements. We will refer to them throughout this article.

\section{Defining discrete and rhythmic movements}

Mathematically, defining rhythmic and discrete movements is an easy task. Rhythmic refers to periodic signals, discrete to aperiodic signals. However, when considering movements that we actually perform, the task becomes more complex, the major problem being that movements are finite in time and that the formal, mathematical definition of periodicity is thus unusable. Moreover, intrinsic variability of movements and modulation by the environment (contacts for instance) change the actual trajectory, so that it is impossible to perform a perfectly periodic trajectory.

The attempt by Hogan and Sternad (2007) to develop a taxonomy to classify discrete and rhythmic movements confirms the inherent difficulty of the task. A discrete movement is defined as a movement which occurs between two postures, where postures stand for a non-zero interval of time where (almost) no movement occurs. Rhythmic movements are categorized in four subsets, going from strictly periodic movements to movements with recurrent patterns. However, as the authors point out in the article, these two definitions are not exclusive. The so-called rhythmic movements occur in between postures (and thus enter the definition of discrete), and discrete movements can be repeated in order to become periodic.

Another difficulty comes from the fact that rhythmic and discrete movements have mainly been studied separately in the literature, although some interesting (relatively recent) articles on their combinations exist (as for instance Hogan and Sternad (2007) or Sternad (2007)). From our point of view, this distinction is mainly due to two interlinked factors. First, rhythmic and discrete movements have not been studied per se in general, but mainly as outcomes of some specific processes in trajectory generation, such as for instance central pattern generators (CPGs) in locomotion and sensorimotor transformations in reaching. Second, studies focusing on the low-level generation of movements often concentrate on rhythmic movements such as locomotion, while those concerning the high-level generation typically address discrete movements such as reaching or grasping. This implies different investigation techniques; most of the studies on rhythmic movements have focused on the spinal cord-brain stem system in deafferented or spinalized subjects, whereas discrete movement is usually studied using brain imaging techniques or kinematic data on awake, behaving animals. Overcoming these differences is a necessary step to understand discrete and rhythmic movements.

These two issues make a review of rhythmic and discrete movements difficult in the sense that any comparison between the numerous studies on the subject is laborious since the methods, the point of view and the physiological level of investigation are different. It is an interesting question whether, in terms of motor control, the apparent differences between discrete and rhythmic movement are artifacts due to different scientific approaches or if both types of movements are in fact produced independently.

Schaal et al (2004) and van Mourik and Beek (2004) for instance have defined three hypotheses that need to be considered: (a) rhythmic movements are repeated discrete movements (concatenation hypothesis), (b) discrete movements correspond to interrupted cyclic 
movements (half-cycle hypothesis) and (c) discrete and rhythmic movements result from different processes (two primitives hypothesis). Note that these three hypotheses would correspond to the One/One case defined above for (a) and (b) and to the Two/Two case for (c). The mixed cases One/Two and Two/One are not considered here as the planning and the execution phase of the movements are not distinguished.

While hypotheses (b) and (c) are still untested, several studies have shown that hypothesis (a) is unlikely to be true. According to van Mourik and Beek (2004), the concatenation hypothesis is mainly a consequence of trajectory planning theory where it is often supposed that discrete segments are used as building blocks for a movement. It has been ruled out by several studies comparing discrete and rhythmic movements (van Mourik and Beek (2004); Hogan and Sternad (2007)), where key kinematic features of rhythmic movements are significantly different from those of discrete movements. Schaal et al (2004) obtained similar results using fMRi techniques: some cortical areas activated during discrete movements where not active during rhythmic ones. In addition, as reported by van Mourik and Beek (2004), Guiard (1993) argued that the concatenation assumption would involved a waste of elastic energy (indeed at the end of a reaching movement, the energy has to be dissipated, whereas for rhythmic movement, the energy can be stored as potential energy for the remaining half-cycle).

It is however important to point out that those comparisons are always made between a reaching movement and its corresponding back and forth rhythmic movements: Thus the difference observed may be due to the characteristics of reaching itself (for instance the control commands required to characterize it) rather than due to the fact that reaching is a discrete movement. For instance, in the experiment conducted by Schaal et al (2004), the subjects had to either cycle around a rest position at a self-chosen amplitude or to stop at a chosen position, to wait for a while and then to start again. fMRI recordings of this experiments have shown that some cortical areas active during the discrete movements were not activated during the rhythmic movements, leading to the conclusion that rhythmic movements cannot be concatenated discrete movements. However, as it has been pointed out, notably by Miall and Ivry (2004), that discrete movements require more processing, namely choosing where to stop and when to start again, which could also explain the differ- ence observed in the fMRI recordings.

Another non negligible phenomenon is the onset and the ending of a rhythmic movement: indeed, boundary conditions change the kinematic properties of the initial and final cycles (compared to normal, in-between cycles), making them closer to those of discrete movements. Indeed, when a discrete movement is performed, the initial and final accelerations are zero while this is not the case during in-between cycles.

van Mourik and Beek (2004) have studied the inbetween cycles and first and last half-cycles separately - They came to the conclusion that, whereas the inbetween cycles were significantly different from the discrete movements, the first and last half cycles were kinematically close to discrete movements. Even if their results do not rule out the half-cycle hypothesis conclusively, they give more support to the two primitives hypothesis: the cyclical movements performed could be in fact a sequence of a discrete, onsetting movement, followed by rhythmic movements and terminated again by a discrete movement. A model by Schöner and Santos (2001) based on this latter hypothesis will be presented in the last part of this review.

The questions on the nature of discrete and rhythmic movements thus remain open, even if strong evidence seems to rule out the concatenation hypothesis. In the next section, we present some work on the interaction of discrete and rhythmic movements in tasks involving their combination.

\section{The combination of discrete and rhythmic movements}

Most of the EMG and kinematic studies on the combination of rhythmic and discrete movements are built on the same scheme: a particular joint (usually the finger or the elbow) has to be moved from an initial to a target position (discrete movement) while oscillating (rhythmic movement). The oscillation is either physiological (Goodman and Kelso (1983); Adamovich et al (1994); Michaels and Bongers (1994); Sternad et al (2000)) or pathological (Wierzbicka et al (1993); Elble et al (1994); Staude et al (2002)), the reader is referred to Sternad (2007) for a thorough review.

In all these experiments, an entrainment effect is observed, that is the discrete movement is phase-coupled with the rhythmic movement, in the sense that the onset of the discrete movement occurs preferably (though 
not always) during a specific phase window of the oscillations. Goodman and Kelso (1983) showed that this phase window corresponds to the peak of momentum of the oscillations in the direction of the discrete movement. Interestingly, it has been shown that professional pistol shooters press the trigger in phase with their involuntary tremor, while beginners try to immobilize themselves before shooting (Tang et al (2008)).

In terms of EMG recordings, the burst initiating the discrete movement occurs approximately at the time where the EMG activity for the rhythmic movement would have been expected without this perturbation. This effect is thus referred to as "burst synchronization" by De Rugy and Sternad (2003). Performing the same experiment, although at different frequencies (lower for De Rugy and Sternad (2003)), Adamovich et al (1994) and De Rugy and Sternad (2003) came to different conclusion on movement combination. Adamovich et al (1994) observed the three following features: (a) the oscillations rapidly attenuate and disappear during the discrete movement and resume after the peak velocity of the discrete movement; (b) there is a phase resetting of the oscillations after the completion of the discrete movement; and (c) the frequency tends to be higher after the discrete movement. In addition, they observed that (d) once the discrete movement is initiated, it is performed independently from the rhythmic one, in the sense that the discrete trajectory is not influenced by the rhythmic movement. Basing themselves on the monotonic hypothesis (St-Onge et al (1993)) according to which the command of the discrete movement stops at the time of its peak velocity, they conclude that discrete and rhythmic movements are excluding each other at the neural level, in the sense that they cannot co-occur. However, their kinematics outcomes outlast them and leads to overlap.

However, Sternad et al (2000) came to a different conclusion concerning the interdependence of the two movements. Indeed, they observed a significant influence of the rhythmic movement on the discrete movement (lower frequencies of oscillations lead to longer discrete movements), which is in contradiction with the result (d) obtained by Adamovich et al (1994). Moreover, the higher frequency observed by Adamovich et al. after a discrete movement (observation (c)) appeared to be a transient phenomenon. Following these observations, Sternad et al (2000) propose that both movements co-occur and that the attenuation of the oscillations during discrete movements is due to inhibitory phenomena.

Note that co-occurrence of discrete and rhythmic movements is supported by a study on whisker movements in rats by Haiss and Schwarz (2005), where it has been found that rhythmic and non rhythmic movements can be evoked through two different areas of the primary motor cortex. It has been shown in addition that simultaneous activation of both areas resulted in a shift of the offset of the whisker oscillations, that is in a combination of both movements. This experiment will be discussed more in details in Section 6.

We now discuss more precisely the generation of discrete and rhythmic movements, both at the execution and at the planning levels.

\section{Generation of discrete and rhythmic movements}

We present movement generation through two fundamental concepts, central pattern generators and force fields, that we develop in the following.

Central pattern generators (CPGs), that is spinal networks involved in many behaviors in vertebrates and invertebrates, is a seminal concept in the generation of (rhythmic) movements (Grillner (1985), Delcomyn (1980)). Although most work on CPGs were originally dedicated to rhythmic movements, Grillner (2006) for instance now extends it to discrete movements as well.

Another important discovery in movement generation is the concept of force fields, which has been brought to light by Bizzi's group (Bizzi et al (1991)). As we will see, force fields provide evidence for a modular organization of the spinal cord circuitry in vertebrates.

In the following we present these two notions in more details, as well as their relationship to discrete and rhythmic movements.

\subsection{Central pattern generators}

Approximatively one century ago, two discrepant explanations for the rhythmic pattern present in locomotion were competing: one suggesting that sensory feedback was the main trigger of the different phases of locomotion (Sherrington (1910)), and another one suggesting the existence of central neural networks capable of generating rhythms without any sensory input (Brown (1912)), such neural networks are now called central pattern generators (CPGs). Brown (1912) showed that cats with transected spinal cord 
and with cut dorsal roots showed rhythmic pattern of muscle activation. Even if, in the initial experiments, the transection of the dorsal roots does not exclude the influence of sensory feedback as pointed out by Grillner and Zangger (1984), there is now very clear evidence that rhythms can be generated centrally without requiring sensory information. Indeed, experiments on lampreys (Cohen and Wallen (1980), Grillner (1985)), on salamanders (Delvolvé et al (1999)) and on frog embryos (Soffe and Roberts (1982)) have shown that when the spinal cord is isolated from the body, electrical or chemical stimulations activate patterns of activity, called fictive locomotion, very similar to the ones observed during intact locomotion. Since then, the CPGs hypothesis has been strengthen by experiments on both vertebrates and invertebrates (see Stein et al (1997) or Ijspeert (2008) for more comprehensive reviews).

Grillner (1985) proposed that CPGs are organized as coupled unit-burst elements with at least one unit per articulation (i.e. per degree of freedom) in the body. Cheng et al (1998) report experiments where these units can be divided even further with independent oscillatory centers for flexor and extensor muscles. Furthermore, several experiments show that CPGs are distributed networks made of multiple coupled oscillatory centers (Ijspeert (2008)).

According to Marder and Bucher (2001), two types of CPG networks can be distinguished: the so-called pacemaker-driven networks and networks with emergent rhythms. Pacemaker-driven networks, which are usually networks that are always active, as in breathing, consist of a subnetwork of intrinsically oscillating neurons that drives non-bursting neurons into a cyclic pattern, while in networks with emergent rhythms, the oscillatory pattern comes from couplings between the neurons, for instance by mutual inhibition of two reciprocal neurons. A mathematical model by Matsuoka (1985) of such a system will be presented in Section 7.

While sensory feedback is not needed for generating the rhythms, it has been shown that some important features of the actual motor pattern are not present in the fictive motor pattern (Stein and Smith (2001)). For instance, in the cat scratching movement, the rhythmic alternation between agonist and antagonist muscles is already present in the fictive motor pattern, whereas the relative duration of extensor activity observed during actual scratching is greater than the one observed in the immobilized preparation (fictive pattern). The motor pattern generated by the CPGs thus seems to be modulated by the sensory-motor information so that it stays coordinated with body movements.

According to Pearson (2000), sensory feedback is also involved in the mechanisms underlying short-term and long-term adaptation of CPGs . He postulates that the long-term phenomena are driven by the body and limb proprioceptors together with central commands and the action of neuromodulators. Kawato (1996) also proposed that persistent errors detected by proprioceptors are used to recalibrate the magnitude of the feed forward command.

In summary, strong evidence for the existence of CPGs in animals exists, as rhythmic patterns of activation were observed both in decerebrated and in deafferented animals, the observed pattern being thus reasonably imputed to the spinal cord alone.

In humans, the activity of the isolated spinal cord is not observable, making the generalization of the previous results difficult: influences from higher cortical areas and from sensory pathways can hardly be excluded (Capaday (2002)). However, evidence suggesting that the spinal cord with intact sensory afferents can generate rhythmic locomotor-like given tonic input is provided by different studies on patients with complete spinal lesion (Dimitrijevic et al (1998)); in addition, Hanna and Frank (1995) reported stepping-like movements in patients before or after brain death and stepping responses have been observed in anencephalic infants just after birth (Peiper and Nagler (1963)). It was shown that treadmill exercises for patients with spinal cord injuries (SCI) improved their walking pattern (Barbeau and Rossignol (1994); Dietz and Harkema (2004); Edgerton et al (2004); Rossignol et al (2007); Wolpaw and Tennissen (2001)) which may be accounted for by the fact that CPGs can be trained to function independently from descending signals (Stein (2008)). Interestingly, Dietz et al (2002) showed that in a setting with $100 \%$ body unloading (thus limiting the role of stretch reflexes), patterned leg movements could be elicited in patients with para- and tetraplegia. Moreover, studies of disabled patients have shown that in the absence of sensory information, gross movement control is preserved, even if peripheral information is necessary for precise movement organization and control (see Jeannerod (1988) or Gandevia and Burke (1992)).

The neonatal stepping movements are an illustration of a complex intra and inter limb coordination of muscle activity, and, even though it lacks some of the unique features of human locomotion, some of its characteristics remain with the onset of real walking, suggesting that the innate pattern could be transformed 
during ontogeny by neural circuits that develop later to obtain mature locomotion (Forssberg (1985)) ${ }^{1}$. Indeed, although the innate stepping response usually (but not always) disappears, the pattern used by toddlers is similar in many aspects to the newborn patterns (Forssberg (1985); Thelen and Cooke (1987). While Forssberg (1985) suggested that the inactive period may be due to a change of excitability in the CPG due to the developing descending locomotor driving signals, Thelen and Cooke (1987) argue that the innate CPGs evolve in a more task specific pattern, notably through the maturation and experience of key subsystems such as balance, posture control and strength.

As mentioned above, most of the early work on CPGs focused on rhythmic movements, but the discovery of functional muscles synergies in the frog linked to discrete movements have led to an extension of the term, as we will see in the next section.

\subsection{Motor primitives and forces fields}

The Bizzi's group provided some evidence for the concept of motor primitives. Indeed, they brought to light that movements were generated in a modular way by the spinal cord in frogs (for a comprehensive review, see Bizzi et al (2008)). More precisely, stimulating specific interneuronal areas of the spinal cord, they observed that the limb was moved in the direction of the same target posture (equilibrium point) whatever the initial position of the limb was. They called the set of the vectors corresponding to the directions obtained by the stimulation force fields. Surprisingly, only 3-4 directions, corresponding to different areas in the spinal cord, were identified (Bizzi et al (1991)); furthermore, they were sufficient to account for natural limb trajectories (Kargo and Giszter (2000)).

Indeed Mussa-Ivaldi et al (1994) found that stimulating two areas simultaneously was almost equivalent to a simple linear combination of the vector of the force fields proportional to the intensity of stimulation. $87.8 \%$ (36 of 41) of the cases could be explained by the summation hypothesis, while an alternative hypothesis, where the outcome correspond to only one of the field (i.e. a winner-take-all approach), was also tested and could explain $58.5 \%$ (24 of 41) of the cases. Under the hypothesis that the fields can be summed, and since

\footnotetext{
${ }^{1}$ It should be however pointed out that the role of transient neonatal reflexes are still unclear, and in particular whether these reflexes are later used to develop mature, voluntary movements or if they correspond to different control levels.
}

the intensity of stimulation does not change the pattern of force orientation (Giszter et al (1993)), the space of possible end-effector target positions could be spanned through the weighted summation of a limited set of force fields. Note that similar results were obtained with rats (Tresch et al (1999)) and cats (Krouchev et al (2006); Ting and Macpherson (2005)).

The co-stimulation assumption supports the hypothesis that movements are produced through the combination of spinal motor primitives which can be characterized by a resulting force field acting on the end-effector of the limb. This seminal result could provide a powerful tool for explaining how the CNS can easily control the many muscles involved in any movement. Indeed, instead of having to activate and control the different muscles involved in the task, the CNS only has to define the level of activation of a small number of synergies. Furthermore, the combination being almost linear, it provides an efficient way of bypassing the inherent nonlinearities present in movement control using direct muscle activation. Tresch et al (1999) have developed a variety of computational methods to extract muscles synergies involved in different movements. Identifying those synergies is a difficult task, mainly because muscles can belong to more than one synergy at a time.

In an experiment using chemical stimulation ${ }^{2}$ (NMDA iontophoresis) of interneurons in the spinal cord of the frog, Saltiel et al (1998) found out that some regions were eliciting rhythmic behaviors. Force measurements of the limb show a finite number of synergies corresponding to the orientation of the oscillations. More precisely, in rhythmic activation, it seems that the equilibrium point changes periodically, leading to an oscillatory behavior. It is thus believed that by stimulating a particular area of the spinal cord, a whole CPG network can be activated thanks to connectivity. Interestingly, the different orientations of oscillation are very close to the direction of the force fields for discrete movements found with the same method. Furthermore, the areas of activation of the discrete and the rhythmic movements for a given orientation were topographically close (Saltiel et al (2005)). This results suggest that rhythms might arise from the temporal combination of simpler discrete modules. According to Saltiel et al (1998), CPGs could be organized such that

\footnotetext{
${ }^{2}$ Although both electrical and chemical microstimulations give the same overall picture for discrete movements (see Saltiel et al (1998)), differences in the typical responses are observed that are due to the fact that electrical microstimulation excites mainly somas and axons, while chemical microstimulation excites dendrites and somas.
} 
the discrete modules provides the orientation of the oscillations while the timing features comes from the network.

It is not known yet if the concept of force fields can be extended to higher vertebrates, but it has been shown that a finite set of (time-variant) synergies of muscles could account for the movement generation in humans during fast reaching movements (d'Avella et al (2006)) as well as in primate grasping (Overduin et al (2008)), providing evidence for the existence of motor primitives.

The difference between discrete and rhythmic movements, at least at the spinal level, may thus be due to differences in the topology ${ }^{3}$ of the network of motor primitives (CPGs, in the broad sense as in Grillner (2006)) rather than to completely distinct pathways. Indeed, discrete networks need to encode a target position and possibly a time of onset, while rhythmic networks also need to be endowed with a notion of frequency and phase. As reviewed by Marder and Bucher (2001), such features seem to emerge naturally from the intrinsic and synaptic properties of the neurons constituting these particular (rhythmic) CPGs.

In summary, there is strong evidence that basic building blocks of movements are present at the spinal level and that they are used by the CNS to create behaviors by combination. However, at this point it is still not clear if distinct motor primitives exist for the generation of discrete and rhythmic movement (One/Two, Two/Two cases) or if discrete and rhythmic movements are generated by the same process (One/One, Two/One cases). It seems reasonable to postulate that the same motor primitives could be involved in the generation of both discrete and rhythmic movements (by specifying target equilibrium points or orientations of oscillations respectively), while features pertaining to rhythmic movements alone (such as frequency and phase) might arise from the coupling properties of the network. In the Section 7, we present a unique dynamical system developed by Degallier et al (2008) that can switch between rhythmic and discrete regimes depending on the input commands.

${ }^{3}$ By network topology, we mean the interconnections between the different elements of the network, including their direction and their types (that is if the connection is excitatory or inhibitory in our case). Indeed, the main point is to consider the behavior emerging from the interactions between the elements (for instance a tonic or an oscillatory output), rather than on the behavior of each element.

\section{Planning of discrete and rhyth- mic movements}

We now address the question of discrete and rhythmic movement during planning. We start by presenting the possible role of motor primitives in movement planning; we then discuss movement encoding by the motor cortex.

\subsection{Motor primitives in movement plan- ning}

A common hypothesis on how we choose to perform a given action is that the CNS uses internal models, that is representations of the sensorimotor system and the environment to select the next action that it is going to produce. An inverse dynamic model is then required for movement initiation, that is to find the activation commands to be sent to the muscles to fulfill the desired task.

The question of how the CNS actually computes the inverse model is still open. Indeed, inverse dynamics problems are complex, in particular in systems with many degrees of freedom, that is with high redundancy. Additionally, the dynamics of the body change with time, as well as external dynamics. According to some authors, the existence of motor primitives might help the CNS to solve the inverse dynamics problem (Bizzi et al (1991); Mussa-Ivaldi (1999); Georgopoulos (1996)). Indeed, motor primitives could provide the CNS with built-in links between muscles and movement direction and hence facilitate the resolution of the inverse problem of finding the muscle commands generating the desired trajectory (Mussa-Ivaldi and Bizzi (2000)).

More precisely, we have seen in Section 5 that motor primitives, at least in frogs, can be combined linearly, bypassing the high nonlinearity of muscles. Thus it can be imagined that instead of solving an inverse problem to control each of the muscles needed to follow the desired trajectory, the CNS chooses a combination of motor primitives that best fits this trajectory. In this case the only task of the CNS is to optimize the activation of each motor primitive in order to minimize the error between the desired and the actual trajectories. According to what was postulated in Section 5, such a hypothesis could mean that discrete movements are represented during planning by the CNS by a (possibly time-varying) equilibrium point in space, whereas 
rhythmic movements would be represented by a (possibly time-varying) direction and a parameter controlling the emerging frequency of oscillation of the network. In both cases the specification of the speed of the movement (or another, related command signal) would also be required to fully determine the movement.

Note that the existence and also the need for internal models is still debated. Basically, the opponents of internal models doubt that the brain is able of imitating the laws of nature, which seems to be required to solve the inverse problem of finding the motor command that gives the desired kinematic outcome (for instance the torque needed to accelerate the end effector of a limb). The reader is referred to articles by Bridgeman (2007) and Feldman (2009) for more details.

We now present some results on movement encoding that are relevant for the control of discrete and rhythmic movements.

\subsection{Movement encoding by the motor cor- tex}

The motor cortex can be subdivided in two areas, the primary motor cortex and the premotor cortex. The latter is formed of the lateral (dorsal and ventral) premotor areas and of the supplementary motor area which are involved in learning sequences of movement, in timing, in the processing of sensorimotor information as well as in the selection of actions.

The primary motor cortex is involved in the control of movement parameters. According to a study by Graziano et al (2002), if the motor cortex is indeed organized somatotopically, it seems that one of the key feature that is encoded in the primary cortex is the location in space towards which the movement is directed. Indeed, in their experiments, regions of the primary motor and premotor cortex of monkeys were stimulated for $500 \mathrm{~ms}$ (the time scale of normal reaching and grasping movements), this duration being longer than in traditional studies. They found that these stimulations were resulting in a complex movement ending in the same location, for any initial position of the limb. They conclude that instead of encoding regions of the body, the motor cortex contains a representation of different complex postures. Note however that these results are still disputed, as reported in Strick (2002), some authors argue that the length of the stimulation and the high currents used do not ensure that only the motor cortex is activated, and thus the resulting movement may be me- diated by other areas than the cortex itself.

Such a finding supports the hypothesis according to which some primary motor cortex neurons are connected in a one-to-one relationship with spinal motor synergies (Ashe (2005)); Georgopoulos (1996) has proposed a model for movement control where levels of activations of motor cortical neurons control the weights of different motor primitives at the spinal level, that is that cortical neurons elicit combinations of preprogrammed basic trajectories rather than encode the complexity of a particular desired trajectory. This could mean that the invariants observed in movement execution are the result of the usage by the CNS of a small set of motor primitives defined at the spinal level rather than a kinematic plan or optimization processes in the supra-spinal structures.

In particular, Haiss and Schwarz (2005) have studied the electric stimulation of different types of whisker movements in the rat, namely rhythmic movement (used for tactile exploration) and whisker retraction (used to sense an object at a specific location). They found that both movements, although performed by the same set of muscles, where elicited by different (but adjacent) regions of the primary motor cortex. Such a result speaks for different representations for discrete and rhythmic movements (Two/One and Two/Two cases), even though it is difficult to conclude at this point whether this is due to the nature of movement (rhythmic or discrete) or simply to the fact that the motor cortex encodes behaviors (as postulated by Graziano et al (2002)). The extension of such an experiment to broader range of movements and animals could possibly provide further insights on the differences between discrete and rhythmic movement generation.

In the same experiment, Haiss and Schwarz (2005) found that stimulating both "discrete" and "rhythmic" areas of the primary motor cortex resulted in a simple combination of the two behaviors: the resulting movement was the oscillation expected when only the rhythmic area is activated, but with an offset corresponding to the discrete movement resulting from the activation of the discrete area. This result is important as it shows that, even if discrete and rhythmic motor primitives result from different processes, which is not established yet, the combination of those primitives still results in a coherent, meaningful behavior. Two models, by De Rugy and Sternad (2003) and Degallier et al (2008), representing complex movements as oscillations around time-varying offset will be presented in the next section. 


\section{Mathematical models for the generation of discrete and rhyth- mic movements}

In this section, we illustrate the four categories (i.e. Two/Two, One/Two, One/One, Two/One) that were defined in Section 2 with six mathematical systems for the generation of discrete and rhythmic movements ${ }^{4}$.

All the mathematical models that we present here are based on dynamical system theory, that is on sets of differential equations that define the evolution of a complex system in time. As we will see, this is a powerful approach to study the qualitative time course of a system as well as the interconnections between its parts ${ }^{5}$.

Furthermore, dynamical systems are particularly well-suited for modeling discrete and rhythmic movements, as among the existing types of stable solutions of a dynamical system - that is, solutions robust against perturbations - two of them correspond to discrete and rhythmic signals: point attractors and limit cycles. Hence a natural solution for modeling discrete and rhythmic motor primitives is to use these stable solutions. Several examples of such modeling are presented in the following.

As a side note, combinations of stable modules are not necessarily stable themselves. However, Slotine and Lohmiller (2001) have shown that a certain form of stability, called contraction ${ }^{6}$, ensures that any combination of such contracting systems is also contracting.

\subsection{Two/Two hypothesis}

In the Two/Two hypothesis, it is assumed that two different, independent processes are involved in the generation of discrete and rhythmic movements. This hypothesis is convenient for modeling, because each process can be optimized in order to finely reproduce the characteristics of both discrete and rhythmic movements. Yet the question of the combination and of the mutual influence of movements is left open.

\footnotetext{
${ }^{4}$ Note that the matlab code used to generate the figures is available at http://biorob2.epfl.ch/users/degallie/bc_matlab.tar.

${ }^{5}$ For an excellent introduction to dynamical systems, see Strogatz (2001).

${ }^{6}$ Contracting systems are defined as nonlinear dynamical systems in which "initial conditions or temporary disturbances are forgotten exponentially fast" (Slotine and Lohmiller (2001), p.138).
}

We start by presenting two independent models for discrete and rhythmic generation, developed by Bullock and Grossberg (1988) and by Matsuoka (1985) respectively. These seminal models, or extensions of them, have been used extensively in the literature (for instance in Schaal et al (2000), De Rugy and Sternad (2003), Degallier et al (2008)).

\section{- The VITE Model: A Neural Command Circuit for Generating Arm and Articulator Trajecto- ries \\ D. Bullock and S. Grossberg, in Dynamic Patterns in Complex Systems, 1988.}

The VITE (Vector Integration To Endpoint) model was originally developed by Bullock and Grossberg (1988) to simulate planned and passive arm movements. The limb position is controlled through a neural command that modifies the respective lengths of a pair of agonist and antagonist muscles according to the desired target position.

The model thus represents a motor primitive that, given a volitional target position, controls in an automatic way a synergy of muscles so that the limb moves to the desired end state. More precisely, here the brain does not encode a trajectory, but a desired state; the actual trajectory emerges from the dynamics of the motor primitive.

The target of the trajectory of each muscle is encoded through a difference vector, i.e. a population of neurons representing the difference between the desired length of the muscle $(T)$ and its actual length $(p)$. The movement is produced by modifying the length of the muscle at a rate $v$ (called the activity) that depends on the difference vector. The whole process is gated by a go command $(G)$ that is a function that can modulate the speed of the movement. There are thus two control parameters, the target length $T$ and the go command $G$, the output of the system being the muscle length $p$. Note that the function $G$ can be chosen to be equal to a constant, a step function or a more complex signal, we will show the impact of the choice of the go command in Fig.3.

Mathematical model. The following set of differential equations generates, for each muscle, a trajectory converging to the target position $T$, at a speed determined by the difference vector $T-p$ and the go command $G$ :

$$
\left\{\begin{array}{l}
\dot{v}=\alpha(T-p-v) \\
\dot{p}=G \max (0, v)
\end{array}\right.
$$


where $\alpha$ is a constant controlling the rate of convergence of the auxiliary variable $v$.
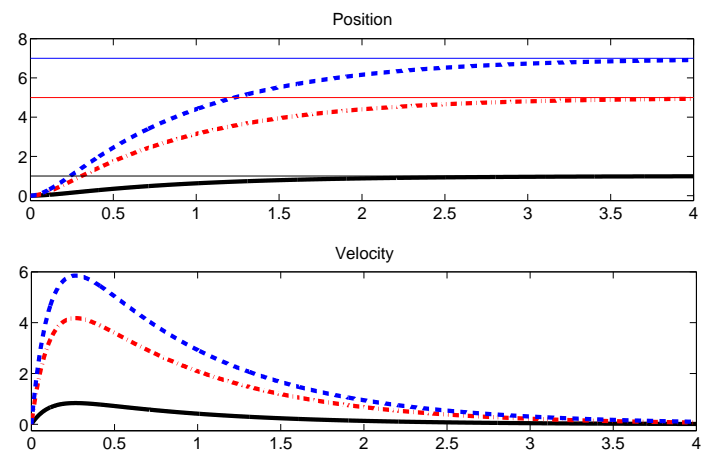

Figure 2: VITE model. Trajectory for three different targets: $T=1$, in black, plain line, $T=5$ in red, dashdotted line and $T=7$ in blue, dotted line). It can be seen that the three trajectories converge to their targets (horizontal lines) at the same time (top graph) and that the velocity peak is proportional to the displacement, i.e. to the difference vector (bottom graph). Here, for all systems, $G=1$ and $\alpha=10$.

As can be seen in the equations, the activity $v$ of the population depends proportionally on the difference vector (the bigger the distance, the higher the activity and thus the speed of contraction of the muscle). In other words, the duration of the movement does not depend on the amount of contraction needed to reach the target length, but is constant, as it is shown in figure 2. Such a feature is very interesting when doing synchronized movements: indeed all the muscles automatically converge to their target length at the same time, whatever the difference between the target and the actual muscle length was. Moreover this system is consistent with the observation that human pointing movements tend to have the same duration, independently of the distance that the hand has to cover (see for instance Morasso (1981)).

The go command $G$ controls both the onset of the movement and its speed profile. Indeed once the target length $T$ is known, nothing prevents the movement from starting but the go command (if it is set to zero). It thus allows movements to be primed before being actually executed. In addition, the amplitude of the go command $G$ allows for a modulation of the speed de-

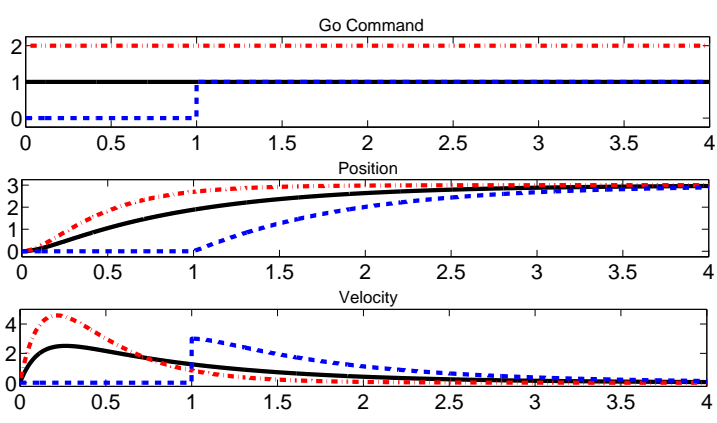

Figure 3: VITE model. Trajectory with three different go commands $G$ : $G=1$, in black, plain line, $G=2$ in red, dash-dotted line and $G=1$ from $t=1 \mathrm{~s}$ and 0 before in blue, dotted line (top graph). For the three systems, the target is constant $(T=3)$. In the middle graph, it is shown that the go command can be used to postpone the onset of the movement and that the duration of the speed of convergence to the target can also be modulated. In the bottom graph, it can be seen that increasing the amplitude of the go command also increases the peak velocity. Here $\alpha=10$.

fined by the difference vector. Thus the CNS can control not only the target of the movement, but also its speed. These features are illustrated in figure 3 with go commands modeled by simple step functions. Note that more complex functions can be chosen as go commands, in order to modify (and in particular smoothen) the velocity profile as will be shown when presenting the model of Degallier et al (2008).

In summary, the VITE model is a very simple model for generating discrete movements with open target position and speed, that allows for synchronized and delayed control of several degrees of freedom. It has been extended many times to different applications, as for instance for visually guided reaching movements (AVITE model, see Gaudiano and Grossberg (1992)) or for modeling the interaction with the spino-muscular system to generate the torque needed to follow a specific trajectory (VITE-FLETE model, see Bullock and Grossberg (1989)).

\section{- Sustained Oscillations Generated by Mutually Inhibiting Neurons with Adaptation \\ K. Matsuoka,}


in Biol. Cybern, 1985.

In this article, Matsuoka (1985) proposes a model for oscillating neural networks. As discussed in section 5, it has been observed that oscillatory behaviors can emerge from networks of mutually inhibiting neurons (see for instance Marder and Bucher (2001)).

In Matsuoka's model, the activity of each neuron is modeled by a simple continuous-variable neuron model originally developed by Morishita and Yajima (1972). An input $S_{i}{ }^{7}$ to the system increases the membrane potential $x_{i}$. When the membrane potential is higher than the threshold value $\theta$, the neuron starts to fire (with firing rate $y_{i}$ ).

Mathematical model. The equations for one neuron are:

$$
\left\{\begin{array}{l}
\dot{x}_{i}=\tau\left(S_{i}-x_{i}\right) \\
\dot{y}_{i}=\max \left(0, x_{i}-\theta\right)
\end{array}\right.
$$

where $\tau$ is a parameter controlling the rate of convergence of $x_{i}$ and $\theta$ is the membrane threshold.

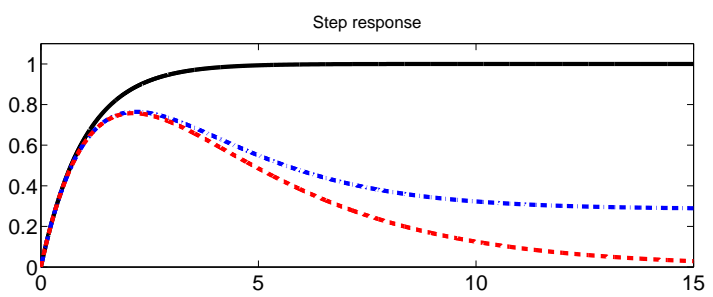

Figure 4: Matsuoka Oscillator. Three typical step responses of a single neuron (i.e. $S_{i}=1$ in each case). In black, plain line, the fatigue parameter $b$ is set to zero (no adaptation) and the output converges monotonically to the input value. In blue, dash-dotted, line $b=2.5$, the output raises but decrease after a while, showing an adaptation effect. Finally in red, dotted line, $b=10$ and it can be seen that the firing rate almost returns to zero (which is the case when $b \rightarrow \infty$ ). In all cases, we used $\tau=1, \theta=0$ and $\tau^{\prime}=12 b / 2.5$ (this value was selected to prevent damped oscillation, see Matsuoka (1985))

In this model, the firing rate increases monotonically and converges to a stationary state, which is not observed in neurons. Matsuoka (1985) thus extends the model to take in account the adaptation $x^{\prime}$ (also called

\footnotetext{
${ }^{7}$ Note that while we take a single value $S_{i}$ as the input to the system, it can be the weighted sum of different inputs.
}

fatigue) of the neurons: when the neuron receives a step input, the firing rate increases rapidly at first and then gradually decreases, as it is shown in Fig.4. Adaptation has indeed been shown to be essential for the generation of oscillations by Reiss (1962) and Suzuki et al (1971).

Mathematical model. The model becomes

$$
\left\{\begin{aligned}
\dot{x_{i}} & =\tau\left(S_{i}-x_{i}-b x_{i}^{\prime}\right) \\
\dot{x}_{i}^{\prime} & =\tau^{\prime}\left(y_{i}-x_{i}^{\prime}\right) \\
\dot{y_{i}} & =\max \left(0, x_{i}-\theta\right)
\end{aligned}\right.
$$

where $\tau^{\prime}(>0)$ and $b(\geq 0)$ control the time course of the adaptation.

The neurons are then coupled to form a network. Here self-inhibition and excitation are not considered.

Mathematical model. For one neuron $j$, the equations are

$$
\left\{\begin{aligned}
\dot{x_{i}} & =S_{i}-x_{i}-b x_{i}^{\prime}-\sum_{j \neq i} a_{i j} y_{j} \\
\dot{x}_{i}^{\prime} & =\tau^{\prime}\left(y_{i}-x_{i}^{\prime}\right) \\
\dot{y_{i}} & =\max \left(0, x_{i}\right)
\end{aligned}\right.
$$

where the $a_{i j}$ 's $(\geq 0)$ are the coupling strengths of the inhibitory connections between neurons $i$ and $j$ and $y_{j}$ is the output of neuron $j$. Note that here, without loss of generality, we take $\theta=0$ and $\tau=1$.

Matsuoka (1985) has derived sufficient conditions for an oscillatory behavior to emerge for different types of networks. The output firing rates for two mutually inhibiting neurons are shown in Fig.5.

Fig. 6 shows two possible oscillating networks of three neurons: one where all the neurons mutually inhibit each other and another one where the neurons unilaterally inhibit each other, that is neuron 1 is for instance only inhibited by neuron 2 and inhibits only neuron 3.

The model offered by Matsuoka is thus a powerful tool to model different oscillatory behaviors. Note that the model can be extended to a muscle command instead of a firing rate as output; we will see an example in the model of De Rugy and Sternad (2003).

Interestingly in this model an oscillatory pattern emerges from the combination of non-cyclic units, thus reproducing the emergent rhythms observed in the spinal cord (see Section 5 for more details). 


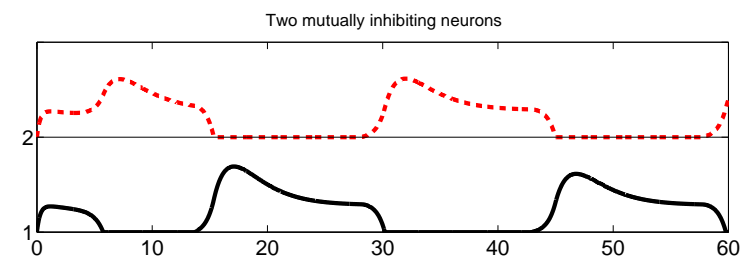

Figure 5: Mastuoka oscillator. The firing rate for two neurons that inhibit each other, with a constant input $S_{i}=1$. Parameters were set to $a_{12}=a_{21}=2.5, \tau=1$, $\theta=0, b=2.5$ and $\tau^{\prime}=12 b / 2.5$

\subsection{One/Two hypothesis}

In the One/Two hypothesis, a similar encoding is used for both discrete and rhythmic movements, that is, there exists a common basic representation for the two types of movements. Such an hypothesis could reflect the analogy observed by Haiss and Schwarz (2005) between the representation of discrete and rhythmic movements in whisker movements in rats (see Section $6)$. In this model, mutual influences of movements are supposed to occur at the muscle level rather than at the spinal level, as discussed above for the Two/Two hypothesis.

We present here the model by Schaal et al (2000), in which both discrete and rhythmic movements are encoded relatively to a difference vector: between the current and desired positions for the discrete movement and between the current and desired amplitudes for the rhythmic movement.

\section{- Nonlinear dynamical systems as movement primitives.}

S. Schaal, S. Kotosaka and D. Sternad, in the proc. of the IEEE International Conference on Humanoid Robotics, 2000

Schaal et al (2000) have developed a model based on the concept of programmable pattern generators (PPGs), that is generators of trajectories with some predefined characteristics and with some open, taskspecific control parameters. Both discrete and rhythmic movements are triggered in a similar way, but they are then generated through different processes. At the end the discrete and the rhythmic output are linearly added to obtain the final trajectory.

In this model, discrete and rhythmic movements are encoded by the difference between the desired state
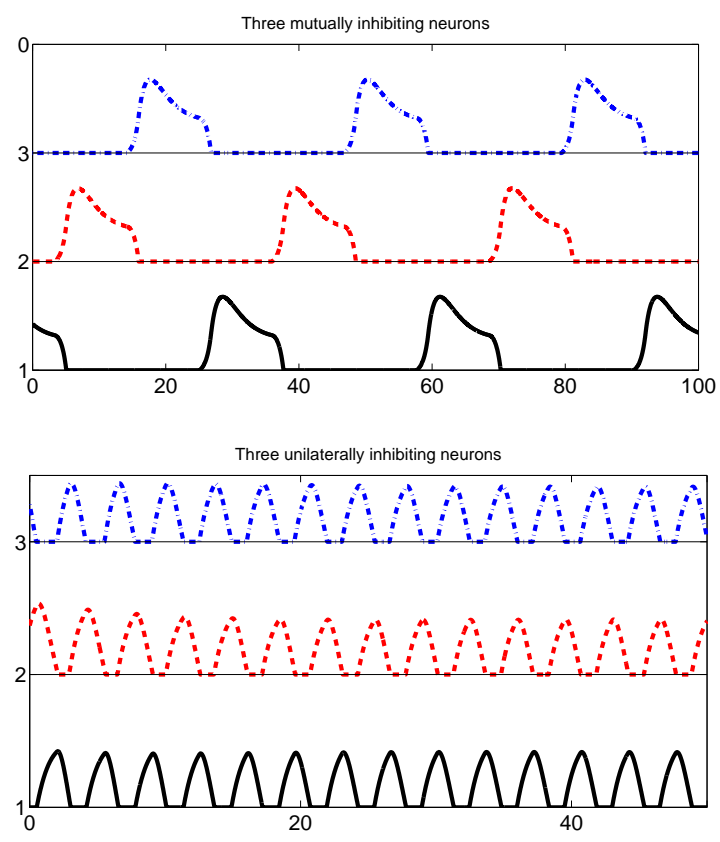

Figure 6: Matsuoka oscillator. The firing rate for two networks of three neurons for a constant input $S_{i}=1$. In the upper graph, the neurons are inhibiting each other, i.e. $a_{i j}=2.5 \forall i, j=1,2,3$. In the second case, the neurons are only unilaterally inhibited, i.e. $a_{12}=a_{23}=$ $a_{30}=2.5$ and $a_{13}=a_{20}=a_{31}=0.0$. Other parameters were set to $a_{21}=2.5$, tau $=1, \theta=0, b=2.5$ and $\tau^{\prime}=$ $12 b / 2.5$

(resp. the position $T$ and the amplitude $A$ ) and the actual state (resp. $p$ and $\theta$ ); the output of the system is the position of the limb $(\alpha=p+\theta)$. This system is quite complex, having many variables and parameters, so that the final output trajectory can be finely tuned to reproduce a desired movement.

The discrete system is a modified version of the VITE model that we have presented before. The movement of the limb is controlled through the speed of contraction of a pair of agonist/antagonist muscles. The difference vector represents the positive difference $\Delta w_{i}$ between the desired target position of the limb $T$ ( $-T$ for the antagonist muscle) and its actual position p. $\Delta w$ is then transformed into an activation pattern $v_{i}$ that resembles what is observed in the primate cortex (see Fig.7, top panel).

Mathematical model. The difference vector for muscle $i$, 

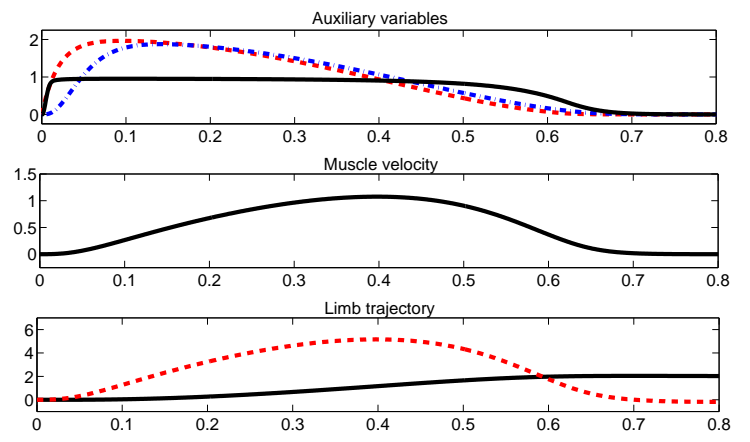

Figure 7: Model by Schaal et al. A typical discrete trajectory converging to the $\operatorname{target} T=1$. On the top panel, the activation pattern is shown in red, dashed line, as well as its smoothened version (in blue, dash-dotted line). The auxiliary variable $r_{i}$, that ensures that the velocity profile is roughly a symmetric, bell-shaped curve, is shown in black, plain line. The middle panel shows the resulting speed $z_{i}$ for the muscle and the bottom panel shows the resulting limb trajectory (in black, plain line) and its speed (red, dashed-line). Here $a_{v}=50.0$, $a_{x}=1, a_{y}=1, a_{r}=50, a_{z}=0.01, a_{p}=0.08, b=10$ and $c_{o}=60$.

$\Delta w_{i}$, is transformed into an activation signal $v_{i}$

$$
\left\{\begin{array}{l}
\Delta w_{i}=\max (0, T-p) \\
\dot{v}_{i}=a_{v}\left(-v_{i}+\Delta w_{i}\right)
\end{array}\right.
$$

where $a_{v}$ is a parameter controlling the rate of convergence of $v_{i}$.

The activation signal is then transformed into a velocity signal $y_{i}$ through a double smoothing. The speed of the movement can be adjusted through the parameter $c_{0}$.

\section{Mathematical model.}

$$
\left\{\begin{array}{l}
\dot{x}_{i}=-a_{x} x_{i}+\left(v_{i}-x_{i}\right) c_{o} \\
\dot{y}_{i}=-a_{y} y_{i}+\left(x_{i}-y_{i}\right) c_{o}
\end{array}\right.
$$

where $a_{y}$ and $a_{x}$ control the rate of convergence of the system and $c_{0}$ controls the speed of the movement.

Finally the velocity $y_{i}$ is integrated in order to obtain the final desired velocity $z_{i}$ for the muscle change (see Fig.7, middle panel). An auxiliary variable $r_{i}$ is used to make $z_{i}$ roughly symmetric and bell-shaped.

\section{Mathematical model.}

$$
\left\{\begin{array}{l}
\dot{r}_{i}=a_{r}\left(-r_{i}+\left(1-r_{i}\right) b v_{i}\right) \\
\dot{z}_{i}=-a_{z} z_{i}+\left(y_{i}-z_{i}\right)\left(1-r_{i}\right) c_{o}
\end{array}\right.
$$

where $a_{r}$ and $b$ control the shape of the signal and are chosen in order to obtain a bell-shaped velocity profile. $a_{z}$ controls the rate of convergence of $z_{i}$.

The velocity commands of the agonist and antagonist muscles $(i$ and $j$ ) are finally integrated to obtain the limb movement $p$ (see Fig.7, bottom panel).

\section{Mathematical model.}

$$
\dot{p}=a_{p}\left(\max \left(0, z_{i}\right)-\max \left(0, z_{j}\right)\right) c_{o}
$$

where $a_{p}$ controls the rate of convergence of the system and $c_{o}$ its speed.

As for the rhythmic movement, it is triggered in a similar way by a difference vector $\Delta \omega_{i}$ between the actual amplitude $\theta$ and the desired amplitude $A . \Delta \omega_{i}$ is turned into an activity signal $\xi_{i}$ (see Fig.8, top panel).

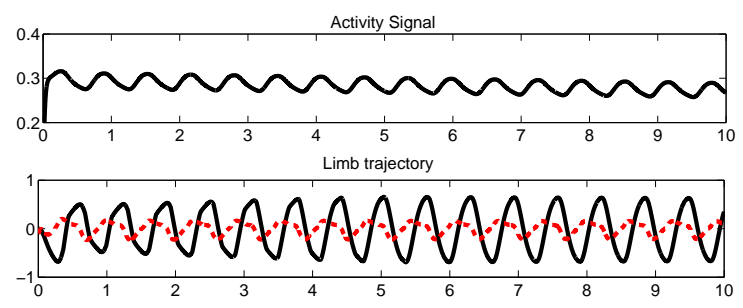

Figure 8: Model by Schaal et al. A typical rhythmic trajectory of amplitude $A=0.6$. The top panel shows the activation pattern $\xi_{i}$. The bottom panel shows the resulting limb trajectory (in black, plain line) and its speed (red, dashed line). Here $a_{\xi}=50.0, a_{\psi}=1.0, \beta=2.5$, $w=2.5$ and $c_{r}=20$.

\section{Mathematical model.}

$$
\left\{\begin{array}{l}
\Delta \omega_{i}=\max (0, A-\theta) \\
\dot{\xi}_{i}=a_{\xi}\left(-\xi_{i}+\Delta \omega_{i}\right)
\end{array}\right.
$$

where $a_{\xi}$ is a parameter controlling the rate of convergence of $\xi_{i}$.

Then, a couple of mutually inhibiting Matsuoka oscillators are used to generate oscillatory velocity signals $\psi_{i}$ and $\psi_{j}$. The oscillator is slightly modified 
to take in account the fact that $\psi_{i}$ represents a velocity and not a position.

\section{Mathematical model.}

$\left\{\begin{array}{l}\dot{\psi}_{i}=-a_{\psi} \psi_{i}+\left(\xi_{i}+\psi_{i}+\beta \zeta_{i}+w \max \left(0, \psi_{j}\right)\right) c_{r} \\ \dot{\zeta}_{i}=-\frac{a_{\psi}}{5} \zeta_{i}+\left(\max \left(0, \psi_{i}\right)-\zeta_{i}\right) \frac{c_{r}}{5}\end{array}\right.$

where $a_{\psi}$ controls the convergence rate of the oscillators and $c_{r}$ the frequency of the oscillations. $w$ controls the strength of the inhibitory coupling.

Finally, the difference between the two oscillators $(i, j)$ is integrated to obtain the desired trajectory $\theta$ (see Fig.7, bottom panel).

\section{Mathematical model.}

$$
\left\{\begin{array}{l}
\dot{\theta}_{i}=\psi_{i} \\
\theta_{r}=c_{r}\left(\max \left(0, \theta_{i}\right)-\max \left(0, \theta_{j}\right)\right)
\end{array}\right.
$$

where $c_{r}$ controls the frequency of the oscillations.

The movement of each degree of freedom is then defined by the linear combination of the output of both signals $(\alpha=p+\theta)$. This linearity allows for a simple, independent control of both movements, but it fails to reproduce the mutual influence of the discrete and rhythmic movements observed in humans.

Note that the primitives can also be coupled together in order to synchronize several degree of freedom during coordinated movement (see Schaal et al (2000) for more details).

The many variable of the model allow for the tuning of desired basic building blocks of movements, yet also makes the system quite complex. It manages to reproduce movements containing many features reminiscent of the human generation of movement, such as a bell-shaped velocity profile for instance.

\subsection{One/One hypothesis}

The One/One hypothesis, that assumes that a unique motor representation and generator are used to produce movements, implies either that one of the movement is a particular case of the other one (i.e. it corresponds, more or less, to the concatenation and half cycle hypotheses mentioned before) or that discrete and rhythmic movement are themselves particular cases of a larger class of movements. The difficulty here is that the model should be designed in order to reproduce the mutual influences observed during movements that are both discrete and rhythmic.

We first present a model that we developed (Degallier et al (2008)), where discrete and rhythmic movement are two particular cases of a larger class of movements. In the second model, by Schöner and Santos (2001), discrete movements are a particular case of rhythmic ones, i.e. discrete movements are considered as truncated rhythmic movements.

\section{- A modular bio-inspired architecture for move- ment generation for the infant-like robot iCub.} S. Degallier, L. Righetti, L. Natale, F. Nori, G. Metta, A.J. Ijspeert,

in the proc. of the second IEEE RAS / EMBS International Conference on Biomedical Robotics and Biomechatronics (BIOROB), 2008.

Degallier et al (2008) present a system where both discrete and rhythmic trajectories are generated through a unique set of differential equations, which is designed to produce complex movements modeled as periodic movements around time-varying offsets. More precisely, the solution of the system can switch between a point attractor and a limit cycle (Hopf bifurcation) depending on one single parameter, so that a unique system can be used for generating both discrete and rhythmic movements.

Here the input is a command specifying the target $T_{i}$ of the discrete movement, and the amplitude $M_{i}$ and frequency $\omega_{i}$ of the rhythmic movement. A zero (or negative) amplitude generates a purely discrete movement and a constant offset generates a purely rhythmic movement. The output of the system is the trajectory of the limb.

The first set of equations controls the discrete movement and is inspired by the VITE model that was presented above. The trajectory converges towards a goal $T_{i}$ and the go command $G_{i}$ is chosen in order to ensure a bell-shaped velocity profile, as illustrated in Fig.9. Similar to the VITE model, all the joints converge synchronously to the target $T_{i}$.

Mathematical model. The discrete primitive, which is inspired from the VITE model, is modeled by the following system of equations

$$
\left\{\begin{array}{l}
\dot{g}_{i}=d\left(p-G_{i}\right) \\
\dot{y}_{i}=G_{i}^{4} v_{i} \\
\dot{v}_{i}=p^{4} \frac{-b^{2}}{4}\left(y_{i}-T_{i}\right)-b v_{i}
\end{array}\right.
$$



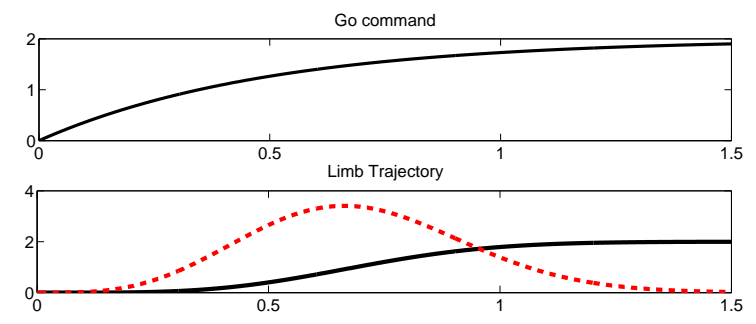

Figure 9: Model by Degallier et al. The top panel shows the go function used in this implementation, that is, a trajectory asymptotically converging to $p_{i}(=2$ here), instead of the step functions presented in the VITE model. Such a go command turns the velocity command into a symmetric, bell-shaped curve (red, dashed line). The black, plain line in the bottom graph shows the resulting limb trajectory converging to the target $T_{i}=2$. Here $d=2$ and $b=2.5$.

The system is critically damped so that the output $y_{i}$ converges asymptotically and monotonically to a goal $T_{i}$ with a speed of convergence controlled by $b$, whereas the speed $v_{i}$ converges to zero. $p$ and $d$ are chosen in order to ensure a bell-shaped velocity profile; $h_{i}$ converges to $p$ and is reset to zero at the end of each movement.

The rhythmic primitive is modeled as a modified Hopf oscillator, which is a simple model that allows for the generation of sinusoidal movements of amplitude $\sqrt{m_{i}}$ and frequency $\omega_{i}$ (that is defined as a combination of the ascending and descending frequencies $\omega_{u p}$ and $\omega_{\text {down }}$, see below). These oscillations can be switched on and off easily through the parameters controlling the amplitude, more precisely, by bifurcation between a limit cycle behavior and a single point attractor.

In this model the expression for the frequency $\omega_{i}$ is slightly modified to allow an independent control of the duration of the ascending $\left(\omega_{u p}\right)$ and descending $\left(\omega_{\text {down }}\right)$ part of the sinusoidal, as illustrated on Fig.10. This feature is particularly useful for independent control of the swing and the stance duration in locomotion.

Mathematical model. The oscillator is governed by the following set of equations

$$
\left\{\begin{array}{l}
\dot{x}_{i}=a\left(M_{i}-r_{i}^{2}\right) x_{i}-\omega_{i} z_{i} \\
\dot{z}_{i}=a\left(M_{i}-r_{i}^{2}\right) z_{i}+\omega_{i} x_{i} \\
\omega_{i}=\frac{\omega_{\text {down }}}{\mathrm{e}^{-f z_{i}}+1}+\frac{\omega_{u p}}{\mathrm{e}^{\mathrm{f} z_{i}}+1}
\end{array}\right.
$$
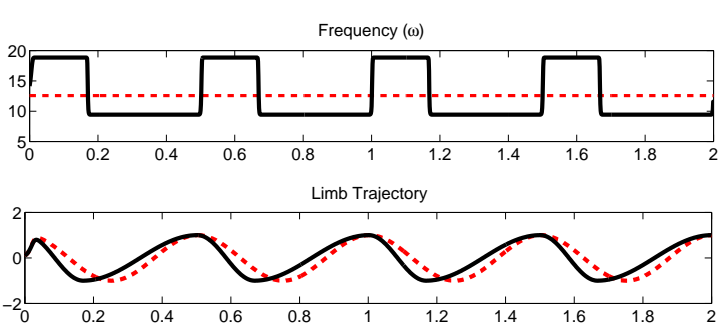

Figure 10: Model by Degallier et al. The top panel shows the value of the frequency $\omega_{i}$ that is modulated through the parameters $\omega_{u p}$ and $\omega_{\text {down }}$. In red, dashed line, $\omega_{u p}=\omega_{\text {down }}$ and the resulting movement (bottom panel) is a normal sinusoidal movement. In black, plain line, $\omega_{\text {down }}>\omega_{\text {up }}$ and the resulting trajectory is a distorted sinusoidal. Note that only $\omega_{\text {down }}$ is controlled, $\omega_{u p}$ being calculated so that $\omega_{i}$ is constant. Here $\omega_{i}=2 \pi$ and $\omega_{\text {down }}=4 \pi$ for the red curve and $\omega_{\text {down }}=6 \pi$ for the black curve. Other parameters are set to $a=100, m=1$ and $f=100$.

where $r_{i}=\sqrt{x_{i}^{2}+z_{i}^{2}}$. $a$ controls the rate of convergence to the limit cycle, $f$ the rapidity of the switching between swing and stance.

The two primitives are then combined together by embedding the discrete movement $y_{i}$ into the rhythmic one as an offset. The system output $x_{i}$ is now an oscillatory movement around a time-varying offset.

Mathematical model. The oscillator is governed by the following set of equations

$$
\left\{\begin{array}{c}
\dot{x}_{i}=a\left(M_{i}-r_{i}^{2}\right)\left(x_{i}-y_{i}\right)-\omega_{i} z_{i} \\
\dot{z}_{i}=a\left(M_{i}-r_{i}^{2}\right) z_{i}+\omega_{i}\left(x_{i}-y_{i}\right)
\end{array}\right.
$$

where now $r_{i}=\sqrt{\left(x_{i}-y_{i}\right)^{2}+z_{i}^{2}}$.

Qualitatively, by simply modifying on the fly the parameters $T_{i}$ and $M_{i}$, the system can switch between purely discrete movements $\left(M_{i}<0, T_{i} \neq\right.$ const), purely rhythmic movements $\left(M_{i}>0, T_{i}=\right.$ const $)$, and combinations of both $\left(M_{i}>0, T_{i} \neq\right.$ const $)$ as illustrated on Fig. 11.

This system allows for a simple modeling of discrete and rhythmic movements. Both dynamics influence each other and, when the movements co-occur, the discrete movement inhibits the rhythmic one, as observed in humans (see Sternad et al (2000), and section 4). 


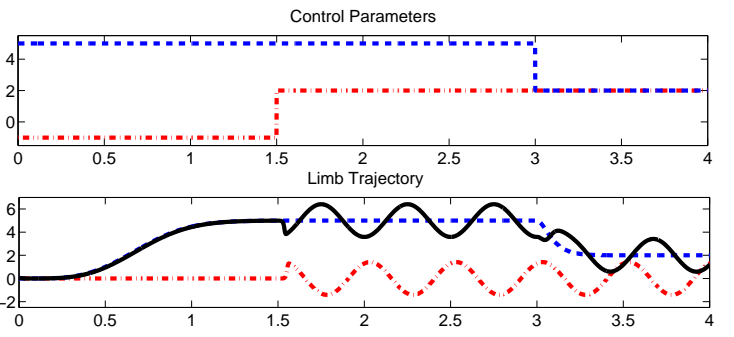

Figure 11: Model by Degallier et al. The top panel shows the target $T_{i}$ for the discrete movement and the resulting trajectory is shown in the bottom panel (in blue, dashed line). In red, dash-dotted line is shown the amplitude control parameter $M_{i}$ (top panel) and the resulting trajectory (bottom panel). In the bottom panel, in black, plain line, the combined trajectory is also shown. Note that it is not a simple linear combination of the discrete and the rhythmic trajectory, which shows the influence of the embedding of the two dynamics. Here omega $_{i}=4 \pi, p_{i}=2, d_{i}=2, b_{i}=2.5, a_{i}=100$ and $f_{i}=100$.

- Control of movement time and sequential action through attractor dynamics: A simulation study demonstrating object interception and coordination.

G. Schöner and C. Santos,

in the proc. of the 9th Intelligent Symposium on Intelligent Robotic Systems, 2001.

The model developed by Schöner and Santos (2001) is built to generate discrete movements, but is based on limit cycles, which makes it easy to extend to the generation of rhythmic movements. Here the input is the target position $T$ of the limb and the output is its trajectory.

In this model, discrete and rhythmic movements are both modeled using limit cycles, i.e. discrete movements are interrupted rhythmic movements. More precisely, here the attractor is a whole trajectory going from the initial position to the target position (contrarily for instance to the VITE model where the trajectory is a transient phenomena and only the target position is a stable attractor). This model can thus successfully explain the observation by Bizzi et al (1984) and Won and Hogan (1995) that when a limb is perturbed during movement execution, it has a tendency to resume the original trajectory, that is it seems that not only the target position matters, but also the trajectory leading to it.

A two-layer system is used, consisting of a layer capable of generating both oscillations and stationary states ("timing layer") and another layer controlling the switching between those states ("neural dynamics control"). The timing layer consists of three terms: the first one is an attractor towards the initial state $x_{i}$, the second one is a Hopf oscillator of amplitude 1 and the third one is an attractor towards the target position $X_{f}$. All these terms are multiplied by the activity level of three "neurons" that are never fully active simultaneously.

Mathematical model. The equations of the timing layer are given by:

$\left\{\begin{array}{l}\dot{x}=-a\left|u_{\mathrm{i}}\right|\left(x-x_{\mathrm{i}}\right)+\left|u_{\mathrm{h}}\right|\left(b\left(1-r^{2}\right) x-\omega y\right)-a\left|u_{\mathrm{f}}\right|\left(x-X_{\mathrm{f}}\right) \\ \dot{y}=-a\left|u_{\mathrm{i}}\right| y+\left|u_{\mathrm{h}}\right|\left(b\left(1-r^{2}\right) y-\omega x\right)-a\left|u_{\mathrm{f}}\right| y\end{array}\right.$

where $x$ is the output of the system and $y$ an auxiliary variable, and $a$ and $b$ are controlling the speed of convergence of the system. In this system, $\left|u_{i}\right|(i=\mathrm{i}, \mathrm{h}, \mathrm{f})$ represents neurons which are never active (i.e. $u_{i}=1$ ) simultaneously.

The sequence of movements is controlled by the neural layer, and more precisely through three neuron activities $u_{i}, u_{h}$ and $u_{f}$ activating the first attractor, the Hopf oscillator and the target attractor respectively - At rest position only the first attractor is active $\left(u_{i}=1, u_{h}=0, u_{f}=0\right)$, so that even if perturbations occur the limb stays at the same position. Then, when a command is received, the Hopf oscillator is activated $\left(u_{h}=1\right)$ and the first attractor deactivated $\left(u_{i}=0\right)$, so that the trajectory follows the limit cycle until it is close enough to the final target. At this moment the Hopf neuron activity $u_{h}$ is set to zero and the final attractor is activated $\left(u_{f}=1\right)$ so that the trajectory converges to the target position $X_{f}$. This sequence of actions is illustrated in Fig.12.

Mathematical model. The timing of activation of the three "neurons" is controlled by the neuronal dynamics which are given by the following equations:

$$
\left\{\begin{array}{l}
\alpha \dot{u}_{\mathrm{i}}=\mu_{\mathrm{i}} u_{\mathrm{i}}-\left|\mu_{\mathrm{i}}\right| u_{\mathrm{i}}^{3}-c\left(u_{\mathrm{h}}^{2}+u_{\mathrm{f}}^{2}\right) u_{\mathrm{i}} \\
\alpha \dot{u}_{\mathrm{h}}=\mu_{\mathrm{h}} u_{\mathrm{h}}-\left|\mu_{\mathrm{h}}\right| u_{\mathrm{h}}^{3}-c\left(u_{\mathrm{i}}^{2}+u_{\mathrm{f}}^{2}\right) u_{\mathrm{h}} \\
\alpha \dot{u}_{\mathrm{f}}=\mu_{\mathrm{f}} u_{\mathrm{f}}-\left|\mu_{\mathrm{f}}\right| u_{\mathrm{f}}^{3}-c\left(u_{\mathrm{i}}^{2}+u_{\mathrm{h}}^{2}\right) u_{\mathrm{f}}
\end{array}\right.
$$

Each equation corresponds to the normal form of a degenerate pitchfork bifurcation controlled by parameters ${ }^{8} \mu_{i}$ with an

\footnotetext{
${ }^{8}$ That is the system has one stable solution $(u=0)$ when $\mu_{i}$ is negative and two stable ones $\left(u_{i}=1\right.$ and $\left.u_{i}=-1\right)$ when $\mu_{i}$ is positive.
} 
extra term to ensure that only one neuron is active, i.e that any solution with more than one neuron active is destabilized. The parameters $\mu_{i}$ are given by

$$
\left\{\begin{array}{l}
\mu_{i}=1.5+2 b_{i} \\
\mu_{h}=1.5+2\left(1-b_{i}\right)\left(1-b_{f}\right) \\
\mu_{f}=1.5+2 b_{f}
\end{array}\right.
$$

where $b_{i}=1$ is equals to 1 when no movement occurs and is set to 0 to activate the movement, and

$$
\left.b_{r}=1-\tanh \left(10\left(0.7 X_{f}-x_{r}(i)\right)\right)+1\right) / 2 .
$$

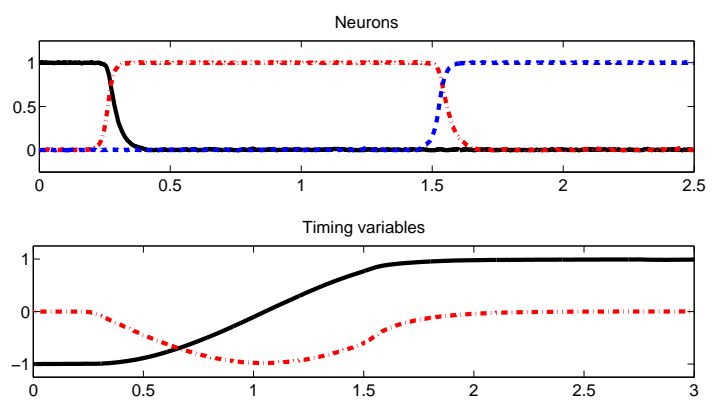

Figure 12: Model by Schoner and Santos. In the top panel, the activity of the three neurons $\left(u_{i}\right.$ in black, plain line, $u_{h}$ in red, dash-dotted line and $u_{f}$ in blue, dashed line) during a typical discrete movement can be observed. Only one neuron is active at a time, corresponding to three stages of the movement: rest at initial position, move to the target and rest at the target position. In the bottom panel, the obtained trajectory $x_{i}$ is shown (in black, plain line) as well as the auxiliary variable $y_{i}$. Here $a=5, b=1, \omega=2, c=2.1$ and $\alpha=0.02$.

Movements can thus be shaped through the neuronal dynamics that qualitatively change the space of solutions of the timing layer. The trajectory in three parts produced by this model (i.e. discrete, rhythmic, discrete) is analogous to the observation by van Mourik and Beek (2004) that the first and last half cycles of a rhythmic movement resemble a discrete movement. In systems with multiple degrees of freedom, coordination can be obtained through the coupling of rhythmic parts of the system (see Schöner and Santos (2001) for more details). Synchronized discrete movements can be obtained through coupling.

\subsection{Two/One hypothesis}

In the Two/One hypothesis, two different motor commands are sent to the same generator. An open question is then how the two motor commands are combined. We present here a model developed by De Rugy and Sternad (2003), initially to explain the phase entrainment effect, where both commands are simply summed.

- Interaction between discrete and rhythmic movements: reaction time and phase of discrete movement initiation during oscillatory movements.

A. de Rugy and D. Sternad,

in Brain Research, 2003

This model has originally been developed to explain the phase entrainment effect observed in humans (please refer to Sec.4 for more details). Here a motor command $S$, composed of the sum of a discrete $S_{d}$ and a rhythmic $S_{r}$ command inputs, is sent to a two-neurons Matsuoka oscillator to generate two firing rates $\left(x_{i}, x_{j}\right)$. These firing rates are then transformed into muscle commands $\left(\mathscr{T}_{i}, \mathscr{T}_{j}\right)$ for a pair of agonist/antagonist muscles and finally to a limb trajectory $\theta$.

The discrete command is modeled as a pulse followed by an exponential decay, resulting in a damped oscillation which, with well-tuned parameters, will later generate a discrete movement. The rhythmic command is simply a constant signal.

Mathematical model. The input command is given by

$$
S=S_{r}+S_{d}
$$

where $S_{r}=$ const and

$$
\dot{S}_{d}=\tau_{s}\left(-S_{d}+p_{d}\right)
$$

where $p_{D}$ is the peak value of the pulse and $\tau_{s}$ a time constant.

A network of two mutually inhibiting Matsuoka oscillators is then used to transform this neural command $S$ into the firing rates $\left(x_{i}, x_{j}\right)$ of two motoneurons controlling a pair of agonist-antagonist muscles.

Mathematical model. The network is governed by the following equations (for one neuron $i$ ):

$$
\left\{\begin{aligned}
\dot{x}_{i} & =\tau_{1}\left(-x_{i}-\beta x_{i}^{\prime}+S-\omega \max \left(0, x_{j}\right)\right) \\
\dot{x}_{i}^{\prime} & =\tau^{\prime}\left(-x_{i}^{\prime}+\max \left(0, x_{i}\right)\right)
\end{aligned}\right.
$$


where $\tau$ and $\tau^{\prime}$ are two parameters controlling the time course of respectively the firing rate $x_{i}$ and the fatigue (or self-inhibition) $x_{i}^{\prime}, \beta$ is the gain of the fatigue component and $x_{j}$ is the output of the second neuron.

The firing rates of the neurons $\left(x_{i}, x_{j}\right)$ are then transformed into torques $\left(\mathscr{T}_{i}, \mathscr{T}_{j}\right)$ exerted by a pair of agonist/antagonist muscles.
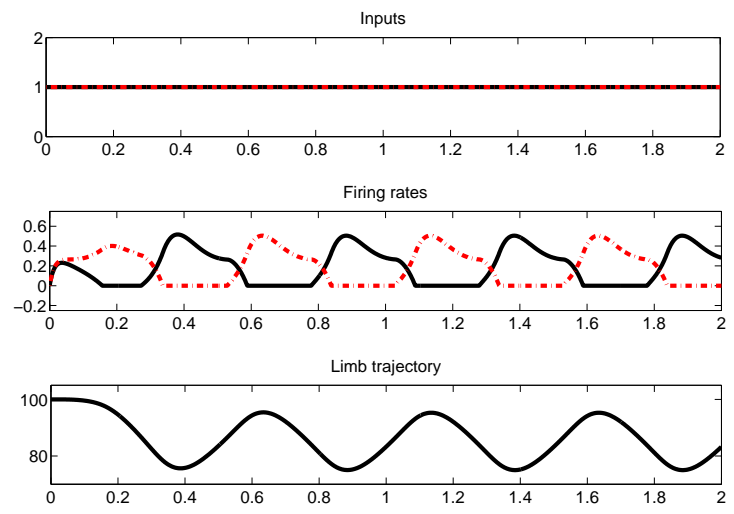

Figure 13: Model by De Rugy and Sternad. A purely rhythmic command $S=S_{R}=1$ (top panel) leads to oscillations of the coupled neurons (middle panel) and the limb (bottom panel). Here $\gamma=0.5, I=0.08, h=5$, $\tau=0.05, \tau^{\prime}=0.125, \tau_{s}=0.2, \beta=2.5$ and $\omega=2.5$.

Mathematical model. The torques are obtained through the following equations:

$$
\left\{\begin{array}{l}
\mathscr{T}_{i}=h_{\mathscr{T}} \max \left(0, x_{i}\right) \\
\mathscr{T}_{j}=-h_{\mathscr{T}} \max \left(0, x_{j}\right)
\end{array}\right.
$$

where $h_{\mathscr{T}}$ is the gain of the torques.

Finally the action of the torques on the movement of the joint $\theta$ is deduced from the dynamics of the limb.

Mathematical model. The dynamics of the limb is governed by the following equation

$$
I \ddot{\theta}+\gamma \dot{\theta}-\left(\mathscr{T}_{i}+\mathscr{T}_{j}\right)=0
$$

where $I$ is the inertia of the limb and $\gamma$ is its damping.

Fig.13 illustrates the output of the model for a rhythmic command (that is, a constant input). The oscillating firing rates are transformed into a smooth, sinusoidal
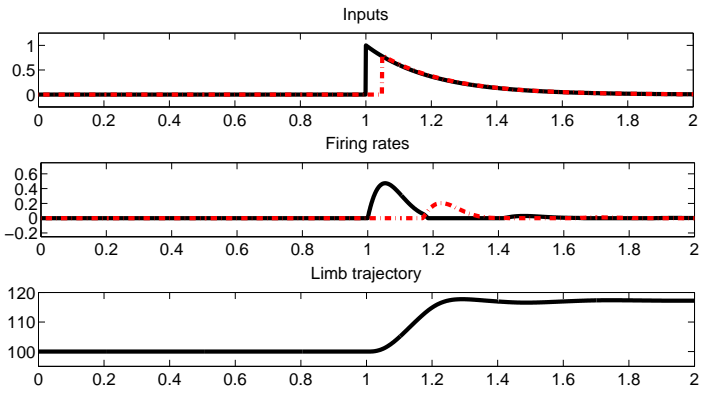

Figure 14: Model by De Rugy and Sternad. A purely discrete command $S=S_{D}$ of peak $p_{D}=1$ (top panel) leads to strongly damped oscillations of the neurons (middle panel), resulting in a discrete movement of the limb (bottom panel). Here $\gamma=0.5, I=0.08, h=5$, $\tau=0.05, \tau^{\prime}=0.125, \tau_{s}=0.2, \beta=2.5$ and $\omega=2.5$.

trajectory through the dynamics of the limb. In Fig.14, it is shown that a purely discrete movement can be obtained using a peak motor command. Finally, in Fig.15, the combination of both command signals and the resulting, combined trajectories are shown.

In this model, there is an entrainment effect that emerges from synchronization effects between the two Matsuoka neurons. The distribution of the offset, as well as the phase lag observed in human subjects were successfully reproduced by this model (De Rugy and Sternad (2003)). Note that this model has been extended by Ronsse et al (2009) to integrate reafferent signals, and thus to capture bimanual features.

\subsection{Discussion of the models}

We have presented different mathematical models, the principle characteristics of which are summarized in Table 1. All these models are based on the concept of motor primitives, that is, simple, non patterned commands from the brain are turned into complex output trajectories governed by the dynamics of the system. So even though the outputs of the models are not at the same representation level, they can easily be modified to account for another level of representation (for instance, De Rugy and Sternad (2003) apply the model of firing rates of neurons of Matsuoka (1985) to limb control by extending the system to the muscles and the limbs dy- 

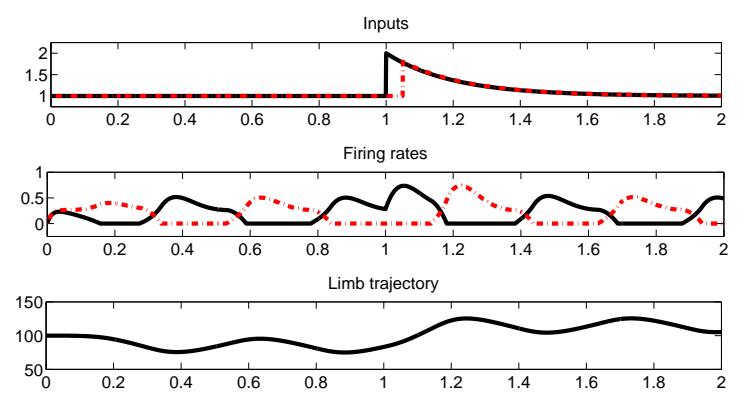

Figure 15: Model by De Rugy and Sternad. A combined command $S=S_{R}+S_{D}$ with $S_{R}=1$ and $p_{D}=1$ (top panel) leads to a perturbed oscillatory behavior of the neurons (middle panel), resulting in a rhythmic movement around a varying offset (bottom panel). Here $\gamma=0.5, I=0.08, h=5, \tau=0.05, \tau^{\prime}=0.125, \tau_{s}=0.2$, $\beta=2.5$ and $\omega=2.5$.

namics).

All these models are successful in producing more or less complex discrete and rhythmic trajectories (except for the models of Matsuoka and Bullock that only model one type of movement). However, in order to be plausible, these models should also be able to reproduce the interaction observed in humans between discrete and rhythmic movements that we have mentioned in Sec.4. As mentioned before, there are two main studies on the subject by Adamovich et al (1994) and Sternad et al (2000), and they come to different conclusions. While they both agree that

(a) the rhythmic movement is inhibited by the discrete one;

(b) the phase of the rhythmic movement is reset after the discrete one;

(c) the frequency tends to be higher after the discrete movement (transient phenomenon according to Sternad et al (2000));

Adamovich et al (1994) conclude that

(d1) the discrete trajectory is not influenced by the rhythmic movement.

which is refuted by Sternad et al (2000), since they observe that

(d2) the rhythmic movement influences the discrete one, or more precisely lower frequencies of oscillations lead to longer discrete movements.

\begin{tabular}{|c|l|c|c|c|c|}
\hline Model & Category & Type & Ctrl & Var & Param \\
\hline \hline Bullocks et al. & Two/Two & $\mathrm{D}$ & 2 & 2 & 1 \\
\hline Matsuoka & Two/Two & $\mathrm{R}$ & $n$ & $3 n$ & $3 n+n(n-1)$ \\
\hline Schaal et al. & One/Two & $\mathrm{D}+\mathrm{R}$ & 2 & 26 & 13 \\
\hline Degallier et al. & One/One & $\mathrm{DR}$ & 3 & 5 & 5 \\
\hline Schoner et al. & One/One & $\mathrm{D} \subset \mathrm{R}$ & 2 & 7 & 8 \\
\hline De Rugy et al. & Two/One & DR & 1 & 5 & 6 \\
\hline
\end{tabular}

Table 1: Main properties of the different models. Type refers to the type of movements and their relationship: $D=$ discrete only, $R=$ rhythmic only, $D+R=$ discrete and rhythmic as a linear combination of the generator outputs, $D R=$ discrete and rhythmic as a unique generator output, $D \subset R=$ discrete as truncated rhythmic. Ctrl is the number of high level commands needed to specify the movement, Var is the number of variables and Param is the number of parameters of the system. For the Matsuoka model, $n$ refers to the number of neurons involved in the network.

To rule out either the Two/Two-One/Two or the One/Two-One/One categories, an efficient way to proceed would be to determine whether the mutual influence between discrete and rhythmic movements appears at the spinal or at the muscular level, i.e. if the discrete and the rhythmic dynamics are influencing each other because there is a unique spinal motor primitive generating them or if it is an artifact due to overlaps during the actual production of the movement. More precisely,

- In both the Two/Two and One/Two hypotheses, the question of the combination of the two movements is left open; more precisely, the interaction has to happen at a lower level of the generation process, that is at the muscular level, as proposed for instance by Adamovich et al (1994) or by Staude et al (2002). Adamovich et al (1994) postulate that discrete and rhythmic movement cannot co-occur, i.e. that any movement can be seen as a sequence of discrete or rhythmic movements. According to them, the mutual influence observed is due to the overlapping of the kinematic outcome of the two movements: they postulate that the kinematic outcome of a movement lasts longer than its generation. Note that this view is not shared by Sternad et al (2000), as was discussed before (see Section 4). Staude et al (2002), for their part, propose that complex movements arise from the summation of the two movements subject to a threshold-linear mechanism; it is interesting 
to note that this simple model manages to model the entrainment effect presented in Sec.4 (please refer to Staude et al (2002) for more details).

- In the One/One hypothesis, the distinction between discrete and rhythmic movements is assumed to be an artifact of movement categorizations, both movements being in fact generated through the same process. In these models, the notion of interaction of the two movements is an ill-posed problem, as they indeed are produced by the same process. In this model, the mechanisms listed above should thus emerge from the dynamics of the system.

○ In the Two/One hypothesis, only the representation of the movements is different, the process generating them being the same. In this case, as in the One/One hypothesis, the observed mutual influence should emerge from the dynamics of the motor primitives, as for instance the entrainment effect in the model by De Rugy and Sternad (2003).

\section{Conclusion}

In this review, we have shown that the concept of motor primitives is an interesting approach to the question of the generation of discrete and rhythmic movements and its modeling, notably through the definition of four categories of models for movement generation illustrated by mathematical models found in the literature. Such categories provide a framework for the analysis of different approaches to the generation of discrete and rhythmic movements and thus to discard or corroborate these approaches.

Since we have chosen to take a functional approach, most of the results that we have presented come from animal studies. Even if these results can not necessarily be generalized to humans in a straightforward way, we believe that they can provide insights into the processes underlying discrete and rhythmic movement generation in humans.

Synergies of muscles have been observed in vertebrates (as reviewed in Sec.5), which indicates that movement may be built through the combination of spinal building blocks of movements that we call motor primitives. Such an assumption has strong implications for the analysis of discrete and rhythmic movements, in the sense that the intrinsic difference between them may lie at the spinal level rather than in the high level commands used to encode them. Indeed, evidence has been presented that both discrete and rhythmic movements could result from spinal motor primitives elicited by simple, non patterned brain commands, suggesting that the two types of movements may simply emerge from a difference in the topologies (oscillatory or not) of the spinal network underlying them.

\section{References}

Adamovich S, Levin M, Feldman A (1994) Merging different motor patterns: Coordination between rhythmical and discrete single-joint movements. Exp Brain Research 99(2):325-337

Ashe $\mathbf{J}$ (2005) What is coded in the primary cortex? In: Riehle A, Vaadia E (eds) Motor Cortex In Voluntary Movements, CRC Press

Barbeau H, Rossignol S (1994) Enhancement of locomotor recovery following spinal cord injury. Curr Opin Neurol 7(6):517-24

Bizzi E, Accornero N, Chapple W, Hogan N (1984) Posture control and trajectory formation during arm movement. J Neurosci 4(11):2738-2744

Bizzi E, Mussa-Ivaldi FA, Giszter S (1991) Computations underlying the execution of movement: a biological perspective. Science 253(5017):287-91

Bizzi E, Cheung VCK, d'Avella A, Saltiel P, Tresch M (2008) Combining modules for movement. Brain Res Rev 57(1):125-33

Bridgeman B (2007) Efference copy and its limitations. Computers in Biology and Medicine 37(7):924-929

Brown T (1912) The factors in rhythmic activity of the nervous system. Proceedings of the Royal Society of London Series 85(579):278-289

Bullock D, Grossberg S (1988) The VITE model: a neural command circuit for generating arm and articulator trajectories. In: Kelso J, Mandell A, Shlesinger M (eds) Dynamic patterns in complex systems, Singapore: World Scientific, pp 206-305

Bullock D, Grossberg S (1989) Volitional Action, Amsterdam:North-Holland, chap VITE and FLETE: Neural Models for trajectory formation and postural control, pp 253-297 
Capaday C (2002) The special nature of human walking and its neural control. TRENDS in Neurosciences 25(7):370-376

Cheng J, Stein R, Jovanovic K, Yoshida K, Bennett D, Han Y (1998) Identification, localization, and modulation of neural networks for walking in the mudpuppy (necturus maculatus) spinal cord. The Journal of Neuroscience 18(11):4295-4304

Cohen A, Wallen P (1980) The neural correlate of locomotion in fish. "fictive swimming" induced in a in vitro preparation of the lamprey spinal cord. Exp Brain Res 41:11-18

d'Avella A, Portone A, Fernandez L, Lacquaniti F (2006) Control of Fast-Reaching movements by muscle synergy combinations. J Neurosci 26(30):77917810

De Rugy A, Sternad D (2003) Interaction between discrete and rhythmic movements: reaction time and phase of discrete movement initiation during oscillatory movements. Brain Research 994(2):160-174

Degallier S, Righetti L, Natale L, Nori F, Metta G, Ijspeert A (2008) A modular bio-inspired architecture for movement generation for the infant-like robot icub. In: Proceedings of the second IEEE RAS / EMBS International Conference on Biomedical Robotics and Biomechatronics, BioRob

Delcomyn F (1980) Neural basis of rhythmic behavior in animals. Science 210:492-498

Delvolvé I, Branchereau P, Dubuc R, Cabelguen JM (1999) Fictive rhythmic motor patterns induced by NMDA in an in vitro brain stem-spinal cord preparation from an adult urodele. Journal of Neurophysiology 82:1074-1077

Dietz V, Harkema SJ (2004) Locomotor activity in spinal cord-injured persons. J Appl Physiol 96(5):1954-1960

Dietz V, Muller R, Colombo G (2002) Locomotor activity in spinal man: significance of afferent input from joint and load receptors. Brain 125(12):2626-2634

Dimitrijevic MR, Gerasimenkp Y, Pinter MM (1998) Evidence for a spinal central pattern generator in humans. Annals of the New York Academy of Sciences 860:360-376
Edgerton VR, Tillakaratne NJ, Bigbee AJ, de Leon RD, Roy RR (2004) Plasticity of the spinal neural circuitry after injury. Annual Review of Neuroscience 27(1):145-167

Elble R, Higgins C, Hughes L (1994) Essential tremor entrains rapid voluntary movements. Exp Neurol 126:138-143

Feldman A (2009) New insights into actionperception coupling. Experimental Brain Research 194(1):3958

Forssberg H (1985) Ontogeny of human locomotor control i. infant stepping, supported locomotion and transition to independent locomotion. Experimental Brain Research 57(3):480-493

Gandevia S, Burke D (1992) Does the nervous system depend on kinesthesic information to control natural limb movements? Behav Brain Sci 15:614-632

Gaudiano P, Grossberg S (1992) Adaptive vector integration to endpoint: Self-organizing neural circuits for control of planned movement trajectories. Human Movement Science 11(1-2):141-155

Georgopoulos AP (1996) On the translation of directional motor cortical commands to activation of muscles via spinal interneuronal systems. Brain Res Cogn Brain Res 3(2):151-5

Giszter SF, Mussa-Ivaldi FA, Bizzi E (1993) Convergent force fields organized in the frog's spinal cord. J Neurosci 13(2):467-91

Goodman D, Kelso J (1983) Exploring the functional signifiance of physiological tremor: A biospectroscopic approach. Experimental Brain Research 49:419-431

Graziano MSA, Taylor CSR, Moore T, Cooke DF (2002) The cortical control of movement revisited. Neuron 36:349-362

Grillner S (1985) Neurobiological bases of rhythmic motor acts in vertebrates. Science 228(4696):143149

Grillner S (2006) Biological pattern generation: The cellular and computational logic of networks in motion. Neuron 52(5):751-766 
Grillner S, Zangger P (1984) The effect of dorsal root transection on the efferent motor pattern in the cat's hindlimb during locomotion. Acta Physiologica Scandinavica 120(3):393-405

Guiard Y (1993) On fittss and hookes laws: Simple harmonic movement in upper-limb cyclical aiming. Acta Psychol (Amst) 82:139-159

Haiss F, Schwarz C (2005) Spatial segregation of different modes of movement control in the whisker representation of rat primary motor cortex. J Neurosci 25(6):1579-87

Hanna JP, Frank JI (1995) Automatic stepping in the pontomedullary stage of central herniation. Neurology 45(5):985-986

Hogan N, Sternad D (2007) On rhythmic and discrete movements: reflections, definitions and implications for motor control. Experimental Brain Research 181(1):13-30

Ijspeert AJ (2008) Central pattern generators for locomotion control in animals and robots: a review. Neural Networks 21(4):642-653

Jeannerod M (1988) The neural and the behavioural organization of goal directed movements. Oxford Science, Oxford

Kandel ER, Schwartz J, Jessell TM (2000) Principles of Neural Science. Mc Graw Hill

Kargo W, Giszter S (2000) Rapid correction of aimed movements by summation of force-field primitives. J Neurosci 20(1):409-426

Kawato M (1996) Learning internal models of the motor apparatus. In: JR Bloedel TESW (ed) The Acquistion of Motor Behavior in Vertebrates, Cambridge MA: MIT Press, pp 409-430

Krouchev N, Kalaska JF, Drew T (2006) Sequential activation of muscle synergies during locomotion in the intact cat as revealed by cluster analysis and direct decomposition. J Neurophysiol 96(4):1991-2010

Marder E, Bucher D (2001) Central pattern generators and the control of rhythmic movements. Curr Biol 11(23):R986-96

Matsuoka K (1985) Sustained oscillations generated by mutually inhibiting neurons with adaptation. Biol Cybern 52:367-376
Miall RC, Ivry R (2004) Moving to a different beat. Nat Neurosci 7(10):1025-6

Michaels C, Bongers R (1994) The dependance of discrete movements on rhythmic movements: simple $\mathrm{rt}$ during oscillatory tracking. Hum Mov Sci 13:473493

Morasso P (1981) Spatial control of arm movements. Experimental Brain Research 42(2):223-227

Morishita I, Yajima A (1972) Analysis and simulation of networks of mutually inhibiting neurons. Biological Cybernetics 11(3):154-165

Mussa-Ivaldi FA (1999) Modular features of motor control and learning. Current Opinion in Neurobiology 9(6):713-717

Mussa-Ivaldi FA, Bizzi E (2000) Motor learning through the combination of primitives. Philos Trans R Soc Lond B Biol Sci 355(1404):1755-69

Mussa-Ivaldi FA, Giszter SF, Bizzi E (1994) Linear combinations of primitives in vertebrate motor control. Proc Natl Ac Sci USA 91:7534-7538

Overduin SA, d'Avella A, Roh J, Bizzi E (2008) Modulation of muscle synergy recruitment in primate grasping. J Neurosci 28(4):880-892

Pearson KG (2000) Neural adaptation in the generation of rhythmic behavior. Ann Rev Physiol 62:723-753

Peiper A, Nagler B (1963) Cerebral Function in Infancy and Childhood. Pitman Medical Publ, London

Reiss RF (1962) A theory and simulation of rhythmic behavior due to reciprocal inhibition in small nerve nets. In: Proceedings of the May 1-3, 1962, spring joint computer conference, ACM, San Francisco, California, pp 171-194

Ronsse R, Sternad D, Lefèvre P (2009) A computational model for rhythmic and discrete movements in uni- and bimanual coordination. Neural Computation 21(5):1335-1370

Rossignol S, Schwab M, Schwartz M, Fehlings MG (2007) Spinal cord injury: Time to move? J Neurosci 27(44):11,782-11,792 
Saltiel P, Tresch MC, Bizzi E (1998) Spinal cord modular organization and rhythm generation: an nmda iontophoretic study in the frog. J Neurophysiol 80(5):2323-39

Saltiel P, Wyler-Duda K, d'Avella A, Ajemian RJ, Bizzi E (2005) Localization and connectivity in spinal interneuronal networks: the adduction-caudal extension-flexion rhythm in the frog. J Neurophysiol 94(3):2120-38

Schaal S, Kotosaka S, Sternad D (2000) Nonlinear dynamical systems as movement primitives. In: International Conference on Humanoid Robotics (Humanoids00), Springer, pp 117-124

Schaal S, Sternad D, Osu R, Kawato M (2004) Rhythmic arm movement is not discrete. Nat Neuroscience 7(10):1136-1143

Schöner G, Santos C (2001) Control of movement time and sequential action through attractor dynamics: A simulation study demonstrating object interception and coordination. In: Neurons, Networks, and Motor Behavior

Sherrington CS (1910) Flexion-reflex of the limb, crossed extension-reflex, and reflex stepping and standing. Journal of Physiology-London 40:28-121

Slotine JJ, Lohmiller W (2001) Modularity, evolution, and the binding problem: a view from stability theory. Neural Netw 14(2):137-45

Soffe S, Roberts A (1982) Tonic and phasic synaptic input to spinal cord motoneurons during fictive locomotion in frog embryos. Journal of Neurophysiology 48(6):1279-1288

St-Onge N, Qi H, Feldman A (1993) The patterns of control signals underlying elbow joint movements in humans. Neurosci Lett 164:171-174

Staude G, Dengler R, Wolf W (2002) The discontinuous nature of motor execution ii. merging discrete and rhythmic movements in a single-joint system the phase entertainment effect. Biological Cybernetics 86(6):427-443

Stein P, Smith J (2001) Neural and biomechanical control strategies for different forms of verterbrates hindlimb motor tasks. In: Stein P, Stuart D, Selverston A (eds) Neurons, Networks and Motor Behavior, Cambridge, MA: MIT press
Stein P, Grillner S, Selverston A, Stuart DE (1997) Neurons, Networks and Motor Behavior. MIT press

Stein RB (2008) The plasticity of the adult spinal cord continues to surprise. The Journal of Physiology 586(12):2823-2823

Sternad D (2007) Rhythmic and discrete movements behavioral, modeling and imaging results. In: Fuchs A, Jirsa V (eds) Coordination Dynamics, Springer

Sternad D, Dean W, Schaal S (2000) Interaction of rhythmic and discrete pattern generators in single joint movements. Hum Mov Science 19:627-665

Strick P (2002) Stimulating research on motor cortex. Nature Neuroscience 5(8):714 - 715

Strogatz SH (2001) Nonlinear Dynamics and Chaos: With Applications to Physics, Biology, Chemistry and Engineering. Perseus Books Group

Suzuki R, Katsuno I, Matano K (1971) Dynamics of neuron ring. Biological Cybernetics 8(1):39-45

Tang W, Zhang W, Huang C, Young M, Hwang I (2008) Postural tremor and control of the upper limb in air pistol shooters. Journal of Sports Sciences 26(14):1579-1587, PMID: 18979336

Thelen E, Cooke DW (1987) Relationship between newborn stepping and later walking: A new interpretation. Developmental Medicine \& Child Neurology 29(3):380-393

Ting LH, Macpherson JM (2005) A limited set of muscle synergies for force control during a postural task. J Neurophysiol 93(1):609-13

Tresch M, Saltiel P, Bizzi E (1999) The construction of movement by the spinal cord. Nature Neuroscience $2: 162-167$

van Mourik AM, Beek PJ (2004) Discrete and cyclical movements: unified dynamics or separate control? Acta Psychol (Amst) 117(2):121-38

Wierzbicka M, Staude G, Wolf W, Dengler R (1993) Relationship between tremor and the onset of rapid voluntary contraction in parkinsons disease. Jour of Neurology, Neurosurgery, and Psychiatry 56:782787 
Wolpaw JR, Tennissen AM (2001) Activity-dependent spinal cord plasticity in health and disease. Annual Review of Neuroscience 24(1):807-843

Won J, Hogan N (1995) Stability properties of human reaching movements. Experimental Brain Research 107(1):125-136 